\title{
Towards the renormalisation of the Standard Model effective field theory to dimension eight: Bosonic interactions I
}

\author{
M. Chala ${ }^{1 *}$, G. Guedes ${ }^{1,2}$, M. Ramos ${ }^{1,2}$, J. Santiago $^{1}$,
}

1 CAFPE and Departamento de Física Teórica y del Cosmos, Universidad de Granada, Campus de Fuentenueva, E-18071 Granada, Spain

2 Laboratório de Instrumentaçao e Física Experimental de Partículas, Departamento de Física da Universidade do Minho, Campus de Gualtar, 4710-057 Braga, Portugal

*mikael.chala@ugr.es

June 22, 2021

\begin{abstract}
We compute the one-loop renormalisation group running of the bosonic Standard Model effective operators to order $v^{4} / \Lambda^{4}$, with $v \sim 246 \mathrm{GeV}$ being the electroweak scale and $\Lambda$ the unknown new physics threshold. We concentrate on the effects triggered by pairs of the leading dimension-six interactions, namely those that can arise at tree level in weakly-coupled ultraviolet completions of the Standard Model. We highlight some interesting consequences, including the interplay between positivity bounds and the form of the anomalous dimensions; the non renormalisation of the $S$ and $U$ parameters; or the importance of radiative corrections to the Higgs potential for the electroweak phase transition. As a byproduct of this work, we provide a complete Green basis of operators involving only the Higgs and derivatives at dimension-eight, comprising 13 redundant interactions.
\end{abstract}

\section{Contents}

16 Introduction 2

\begin{tabular}{|lll}
\hline 2 & Theory and conventions & 3
\end{tabular}

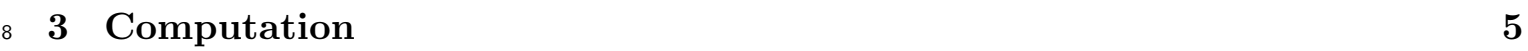

\begin{tabular}{|lll}
\hline 4 & The structure of the renormalisation group equations & 9
\end{tabular}

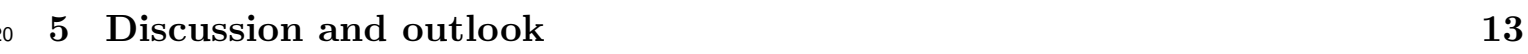

${ }_{21}$ A Removing redundant operators 16

$\begin{array}{lll}22 & \text { B Renormalisation group equations } & 18\end{array}$

${ }_{23}^{23}$ C Ultraviolet completion of the Standard Model 22

24 References $\quad 23$ 


\section{Introduction}

The Standard Model (SM) extended with effective interactions, also known as SM effective field theory (SMEFT) [1, is increasingly becoming one of the favourite options for describing particle physics at currently explored energies. The main reasons are the apparent absence of new resonances below the $\mathrm{TeV}$ scale $[2]$ and the fact that in general the SMEFT explains the experimental data better than the SM alone [3].

Relatively to the SM, the impact of effective operators of dimension $d>4$ on observables computed at energy $\sim E$ is of order $(E / \Lambda)^{d-4}$, with $\Lambda \gg E$ being the (unknown) new physics threshold. Thus, the most relevant interactions are those of lowest energy dimension, which, ignoring lepton number violation (LNV), are the ones of dimension six. These operators have been experimentally tested from very different angles at all kind of particle physics facilities. In particular, the knowledge of the corresponding renormalisation group running $4-10$ has allowed the high-energy physics community to probe the SMEFT to order $E^{2} / \Lambda^{2}$ combining experimental information gathered across very different energies; see for example Refs. [11 14].

However, there is by now convincing evidence that dimension-six operators do not suffice for making predictions within the SMEFT in a number of situations. For example, dimension-six interactions do not provide the dominant contribution to some observables [15] or even they do not arise at tree level in concrete ultraviolet (UV) completions of the SM [16, 17]. It can be also that relatively low values of $\Lambda$ are favoured by data in some interactions, and therefore corrections involving higher powers of $E / \Lambda$ are not negligible 16, 18; or simply that some observables are so well measured (or constrained) that they are sensitive to higher-dimensional operators 18 20.

In either case, dimension-eight operators must be retained when using the SMEFT. (Dimension-seven interactions [21,22] are also LNV.) This has been in fact the approach adopted in a number of recent theoretical works [16, 18 20, 23 28], but so far mostly at tree level. Our goal is to make a first step forward towards the renormalisation of the SMEFT to order $E^{4} / \Lambda^{4}$. We think that, beyond opening the door to using the SMEFT precisely and consistently across energy scales, there are several motivations to address this challenge. For example:

1. Several classes of dimension-eight operators (including purely bosonic) that arise only at one loop in weakly-coupled UV completions of the SM can be renormalised by dimension-eight terms that can be generated at tree level [29]. (While at dimension six this occurs solely in one case.) This implies that the running of some operators can provide the leading SMEFT corrections to SM predictions in observables in which only loop-induced interactions contribute at tree level.

2. Eight is the lowest dimension at which there exist two co-leading contributions to renormalisation within the SMEFT: one involving single insertions of dimension-eight operators, and another one consisting of pairs of dimension-six interactions. (Pairs of dimension-five operators renormalise dimension-six ones [30], but they are LNV and therefore sub-leading with respect to single dimension-six terms.) Non-renormalisation theorems have been established only in relation to the first contribution [17,29. Thus, whether tree-level dimension-six operators renormalise loop-induced dimension-eight interactions is, to the best of our knowledge, still unknown. 
3. Dimension-eight operators are subject to positivity bounds [31 37]. Thus, precisely because dimension-six interactions mix into dimension-eight ones, it is a priori conceivable that theoretical constraints on combinations of dimension-six Wilson coefficients can be established if the corresponding renormalisation group equations (RGEs) are precisely known.

Inspired by these observations, and in particular by $\mathbf{2}$, in this paper we will focus on renormalisation triggered by dimension-six operators. (We will consider the effects of higher-dimensional operators in loops in subsequent works.) Also, we will concentrate on the running of the bosonic sector of the SMEFT.

This article is organised as follows. In section 2 we introduce the relevant Lagrangian and clarify the notation used thorough the rest of the paper. In section 3 we describe the technical details of the renormalisation programme. In section 4 we unravel the global structure of the renormalisation group equations (RGEs). We finalise with a discussion of the results in section 5. We dedicate Appendix A to relations that hold on-shell between different operators. In Appendix B we write explicitly all RGEs, while in Appendix C we describe briefly a UV model that accounts for generic tree-level generated dimension-six bosonic operators.

\section{Theory and conventions}

We denote by $e, u$ and $d$ the right-handed (RH) leptons and quarks; while $l$ and $q$ refer to the left-handed ( $\mathrm{LH})$ counterparts. The electroweak (EW) gauge bosons and the gluon are named by $W, B$ and by $G$, respectively. We represent the Higgs doublet by $\phi=\left(\phi^{+}, \phi^{0}\right)^{T}$, and $\tilde{\phi}=\mathrm{i} \sigma_{2} \phi^{*}$ with $\sigma_{I}(I=1,2,3)$ being the Pauli matrices. Thus, the renormalisable SM Lagrangian reads:

$$
\begin{aligned}
\mathcal{L}_{\mathrm{SM}}= & -\frac{1}{4} G_{\mu \nu}^{A} G^{A \mu \nu}-\frac{1}{4} W_{\mu \nu}^{a} W^{a \mu \nu}-\frac{1}{4} B_{\mu \nu} B^{\mu \nu} \\
& +\overline{q_{L}^{\alpha}} \mathrm{i} \not D q_{L}^{\alpha}+\overline{l_{L}^{\alpha}} \mathrm{i} \not D l_{L}^{\alpha}+\overline{u_{R}^{\alpha}} \mathrm{i} \not D u_{R}^{\alpha}+\overline{d_{R}^{\alpha}} \mathrm{i} \not D d_{R}^{\alpha}+\overline{e_{R}^{\alpha}} \mathrm{i} \not D e_{R}^{\alpha} \\
& +\left(D_{\mu} \phi\right)^{\dagger}\left(D^{\mu} \phi\right)-\mu^{2}|\phi|^{2}-\lambda|\phi|^{4}-\left(y_{\alpha \beta}^{u} \overline{q_{L}^{\alpha}} \widetilde{\phi} u_{R}^{\beta}+y_{\alpha \beta}^{d} \overline{q_{L}^{\alpha}} \phi d_{R}^{\beta}+y_{\alpha \beta}^{e} \overline{l_{L}^{\alpha}} \phi e_{R}^{\beta}+\text { h.c. }\right) .
\end{aligned}
$$

We adopt the minus-sign convention for the covariant derivative:

$$
D_{\mu}=\partial_{\mu}-\mathrm{i} g_{1} Y B_{\mu}-i g_{2} \frac{\sigma^{I}}{2} W_{\mu}^{I}-\mathrm{i} g_{3} \frac{\lambda^{A}}{2} G_{\mu}^{A},
$$

where $g_{1}, g_{2}$ and $g_{3}$ represent, respectively, the $U(1)_{Y}, S U(2)_{L}$ and $S U(3)_{c}$ gauge couplings, $Y$ stands for the hypercharge and $\lambda^{A}$ are the Gell-Mann matrices.

We use the Warsaw basis 38 for the dimension-six SMEFT Lagrangian $\mathcal{L}^{(6)}$, and the basis of Ref. 17 for the dimension-eight part $\mathcal{L}^{(8)}$. (An equivalent basis can be found in Ref. [39].) While the renormalisation of $\mathcal{L}^{(6)}$ has been studied at length $\left.[7] 9\right]$, the running of $\mathcal{L}^{(8)}$ is largely unknown. Assuming lepton-number conservation, the running of dimension-eight Wilson coefficients receives contributions from loops involving single insertions of dimension-eight couplings as well as from pairs of dimension-six operators. Schematically:

$$
16 \pi^{2} \mu \frac{d c_{i}^{(8)}}{d \mu}=\gamma_{i j} c_{j}^{(8)}+\gamma_{i j k}^{\prime} c_{j}^{(6)} c_{k}^{(6)} .
$$

Although $c^{(6)}$ (and $c^{(8)}$ ) are in general unknown, fits of the SMEFT to the data favour relatively large values of some of these coefficients [3]. This implies that the $\gamma^{\prime}$ term, which 


\begin{tabular}{|c|c|c|c|c|}
\hline$\stackrel{\infty}{\theta}$ & $\begin{array}{l}\text { Operator } \\
\qquad\left(\phi^{\dagger} \phi\right)^{4}\end{array}$ & $\begin{array}{l}\text { Notation } \\
\mathcal{O}_{\phi^{8}}\end{array}$ & Operator & Notation \\
\hline $\begin{array}{l}\tilde{a} \\
\hat{6} \\
0\end{array}$ & $\left(\phi^{\dagger} \phi\right)^{2}\left(D_{\mu} \phi^{\dagger} D^{\mu} \phi\right)$ & $\mathcal{O}_{\phi^{6}}^{(1)}$ & $\left(\phi^{\dagger} \phi\right)\left(\phi^{\dagger} \sigma^{I} \phi\right)\left(D_{\mu} \phi^{\dagger} \sigma^{I} D^{\mu} \phi\right)$ & $\mathcal{O}_{\phi^{6}}^{(2)}$ \\
\hline \multirow{2}{*}{$\underset{A}{+1}$} & $\left(D_{\mu} \phi^{\dagger} D_{\nu} \phi\right)\left(D^{\nu} \phi^{\dagger} D^{\mu} \phi\right)$ & $\mathcal{O}_{\phi^{4}}^{(1)}$ & $\left(D_{\mu} \phi^{\dagger} D_{\nu} \phi\right)\left(D^{\mu} \phi^{\dagger} D^{\nu} \phi\right)$ & $\mathcal{O}_{\phi^{4}}^{(2)}$ \\
\hline & $\left(D^{\mu} \phi^{\dagger} D_{\mu} \phi\right)\left(D^{\nu} \phi^{\dagger} D_{\nu} \phi\right)$ & $\mathcal{O}_{\phi^{4}}^{(3)}$ & & \\
\hline \multirow{2}{*}{ 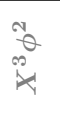 } & $\epsilon^{I J K}\left(\phi^{\dagger} \phi\right) W_{\mu}^{I \nu} W_{\nu}^{J \rho} W_{\rho}^{K \mu}$ & $\mathcal{O}_{W^{3} \phi^{2}}^{(1)}$ & $\epsilon^{I J K}\left(\phi^{\dagger} \phi\right) W_{\mu}^{I \nu} W_{\nu}^{J \rho} \widetilde{W}_{\rho}^{K \mu}$ & $\mathcal{O}_{W^{3} \phi^{2}}^{(2)}$ \\
\hline & $\epsilon^{I J K}\left(\phi^{\dagger} \sigma^{I} \phi\right) B_{\mu}^{\nu} W_{\nu}^{J \rho} W_{\rho}^{K \mu}$ & $\mathcal{O}_{W^{2} B \phi^{2}}^{(1)}$ & $\epsilon^{I J K}\left(\phi^{\dagger} \sigma^{I} \phi\right)\left(\widetilde{B}^{\mu \nu} W_{\nu \rho}^{J} W_{\mu}^{K \rho}+B^{\mu \nu} W_{\nu \rho}^{J} \widetilde{W}_{\mu}^{K \rho}\right)$ & $\mathcal{O}_{W^{2} B \phi^{2}}^{(2)}$ \\
\hline \multirow{5}{*}{ 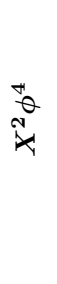 } & $\left(\phi^{\dagger} \phi\right)^{2} G_{\mu \nu}^{A} G^{A \mu \nu}$ & $O_{G^{2} \phi^{4}}^{(1)}$ & $\left(\phi^{\dagger} \phi\right)^{2} \widetilde{G}_{\mu \nu}^{A} G^{A \mu \nu}$ & $O_{G^{2} \phi^{4}}^{(2)}$ \\
\hline & $\left(\phi^{\dagger} \phi\right)^{2} W_{\mu \nu}^{I} W^{I \mu \nu}$ & $\mathcal{O}_{W^{2} \phi^{4}}^{(1)}$ & $\left(\phi^{\dagger} \phi\right)^{2} \widetilde{W}_{\mu \nu}^{I} W^{I \mu \nu}$ & $\mathcal{O}_{W^{2} \phi^{4}}^{(2)}$ \\
\hline & $\left(\phi^{\dagger} \sigma^{I} \phi\right)\left(\phi^{\dagger} \sigma^{J} \phi\right) W_{\mu \nu}^{I} W^{J \mu \nu}$ & $\mathcal{O}_{W^{2} \phi^{4}}^{(3)}$ & $\left(\phi^{\dagger} \sigma^{I} \phi\right)\left(\phi^{\dagger} \sigma^{J} \phi\right) \widetilde{W}_{\mu \nu}^{I} W^{J \mu \nu}$ & $\mathcal{O}_{W^{2} \phi^{4}}^{(4)}$ \\
\hline & $\left(\phi^{\dagger} \phi\right)\left(\phi^{\dagger} \sigma^{I} \phi\right) W_{\mu \nu}^{I} B^{\mu \nu}$ & $\mathcal{O}_{W B \phi^{4}}^{(1)}$ & $\left(\phi^{\dagger} \phi\right)\left(\phi^{\dagger} \sigma^{I} \phi\right) \widetilde{W}_{\mu \nu}^{I} B^{\mu \nu}$ & $\mathcal{O}_{W B \phi^{4}}^{(2)}$ \\
\hline & $\left(\phi^{\dagger} \phi\right)^{2} B_{\mu \nu} B^{\mu \nu}$ & $\mathcal{O}_{B^{2} \phi^{4}}^{(1)}$ & $\left(\phi^{\dagger} \phi\right)^{2} \widetilde{B}_{\mu \nu} B^{\mu \nu}$ & $\mathcal{O}_{B^{2} \phi^{4}}^{(2)}$ \\
\hline \multirow{8}{*}{ 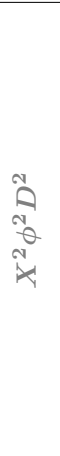 } & $\left(D^{\mu} \phi^{\dagger} D^{\nu} \phi\right) W_{\mu \rho}^{I} W_{\nu}^{I \rho}$ & $\mathcal{O}_{W^{2} \phi^{2} D^{2}}^{(1)}$ & $\left(D^{\mu} \phi^{\dagger} D_{\mu} \phi\right) W_{\nu \rho}^{I} W^{I \nu \rho}$ & $\mathcal{O}_{W^{2} \phi^{2} D^{2}}^{(2)}$ \\
\hline & $\left(D^{\mu} \phi^{\dagger} D_{\mu} \phi\right) W_{\nu \rho}^{I} \widetilde{W}^{I \nu \rho}$ & $\mathcal{O}_{W^{2} \phi^{2} D^{2}}^{(3)}$ & $i \epsilon^{I J K}\left(D^{\mu} \phi^{\dagger} \sigma^{I} D^{\nu} \phi\right) W_{\mu \rho}^{J} W_{\nu}^{K \rho}$ & $\mathcal{O}_{W^{2} \phi^{2} D^{2}}^{(4)}$ \\
\hline & $\epsilon^{I J K}\left(D^{\mu} \phi^{\dagger} \sigma^{I} D^{\nu} \phi\right)\left(W_{\mu \rho}^{J} \widetilde{W}_{\nu}^{K \rho}-\widetilde{W}_{\mu \rho}^{J} W_{\nu}^{K \rho}\right)$ & $\mathcal{O}_{W^{2} \phi^{2} D^{2}}^{(5)}$ & $i \epsilon^{I J K}\left(D^{\mu} \phi^{\dagger} \sigma^{I} D^{\nu} \phi\right)\left(W_{\mu \rho}^{J} \widetilde{W}_{\nu}^{K \rho}+\widetilde{W}_{\mu \rho}^{J} W_{\nu}^{K \rho}\right)$ & $\mathcal{O}_{W^{2} \phi^{2} D^{2}}^{(6)}$ \\
\hline & $\left(D^{\mu} \phi^{\dagger} \sigma^{I} D_{\mu} \phi\right) B_{\nu \rho} W^{I \nu \rho}$ & $\mathcal{O}_{W B \phi^{2} D^{2}}^{(1)}$ & $\left(D^{\mu} \phi^{\dagger} \sigma^{I} D_{\mu} \phi\right) B_{\nu \rho} \widetilde{W}^{I \nu \rho}$ & $\mathcal{O}_{W B \phi^{2} D^{2}}^{(2)}$ \\
\hline & $i\left(D^{\mu} \phi^{\dagger} \sigma^{I} D^{\nu} \phi\right)\left(B_{\mu \rho} W_{\nu}^{I \rho}-B_{\nu \rho} W_{\mu}^{I \rho}\right)$ & $\mathcal{O}_{W B \phi^{2} D^{2}}^{(3)}$ & $\left(D^{\mu} \phi^{\dagger} \sigma^{I} D^{\nu} \phi\right)\left(B_{\mu \rho} W_{\nu}^{I \rho}+B_{\nu \rho} W_{\mu}^{I \rho}\right)$ & $\mathcal{O}_{W B \phi^{2} D^{2}}^{(4)}$ \\
\hline & $i\left(D^{\mu} \phi^{\dagger} \sigma^{I} D^{\nu} \phi\right)\left(B_{\mu \rho} \widetilde{W}_{\nu}^{I} \rho-B_{\nu \rho} \widetilde{W}_{\mu}^{I} \rho\right)$ & $\mathcal{O}_{W B \phi^{2} D^{2}}^{(5)}$ & $\left(D^{\mu} \phi^{\dagger} \sigma^{I} D^{\nu} \phi\right)\left(B_{\mu \rho} \widetilde{W}_{\nu}^{I} \rho+B_{\nu \rho} \widetilde{W}_{\mu}^{I} \rho\right)$ & $\mathcal{O}_{W B \phi^{2} D^{2}}^{(6)}$ \\
\hline & $\left(D^{\mu} \phi^{\dagger} D^{\nu} \phi\right) B_{\mu \rho} B_{\nu}^{\rho}$ & $\mathcal{O}_{B^{2} \phi^{2} D^{2}}^{(1)}$ & $\left(D^{\mu} \phi^{\dagger} D_{\mu} \phi\right) B_{\nu \rho} B^{\nu \rho}$ & $\mathcal{O}_{B^{2} \phi^{2} D^{2}}^{(2)}$ \\
\hline & $\left(D^{\mu} \phi^{\dagger} D_{\mu} \phi\right) B_{\nu \rho} \widetilde{B}^{\nu \rho}$ & $\mathcal{O}_{B^{2} \phi^{2} D^{2}}^{(3)}$ & & \\
\hline \multirow{3}{*}{ 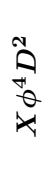 } & $\mathrm{i}\left(\phi^{\dagger} \phi\right)\left(D^{\mu} \phi^{\dagger} \sigma^{I} D^{\nu} \phi\right) W_{\mu \nu}^{I}$ & $\mathcal{O}_{W \phi^{4} D^{2}}^{(1)}$ & $\mathrm{i}\left(\phi^{\dagger} \phi\right)\left(D^{\mu} \phi^{\dagger} \sigma^{I} D^{\nu} \phi\right) \widetilde{W}_{\mu \nu}^{I}$ & $\mathcal{O}_{W \phi^{4} D^{2}}^{(2)}$ \\
\hline & $\mathrm{i} \epsilon^{I J K}\left(\phi^{\dagger} \sigma^{I} \phi\right)\left(D^{\mu} \phi^{\dagger} \sigma^{J} D^{\nu} \phi\right) W_{\mu \nu}^{K}$ & $\mathcal{O}_{W \phi^{4} D^{2}}^{(3)}$ & $\mathrm{i} \epsilon^{I J K}\left(\phi^{\dagger} \sigma^{I} \phi\right)\left(D^{\mu} \phi^{\dagger} \sigma^{J} D^{\nu} \phi\right) \widetilde{W}_{\mu \nu}^{K}$ & $\mathcal{O}_{W \phi^{4} D^{2}}^{(4)}$ \\
\hline & $\mathrm{i}\left(\phi^{\dagger} \phi\right)\left(D^{\mu} \phi^{\dagger} D^{\nu} \phi\right) B_{\mu \nu}$ & $\mathcal{O}_{B \phi^{4} D^{2}}^{(1)}$ & $\mathrm{i}\left(\phi^{\dagger} \phi\right)\left(D^{\mu} \phi^{\dagger} D^{\nu} \phi\right) \widetilde{B}_{\mu \nu}$ & $\mathcal{O}_{B \phi^{4} D^{2}}^{(2)}$ \\
\hline
\end{tabular}

Table 1: Basis of bosonic dimension-eight operators involving the Higgs. We follow the notation from Ref. [17]. All interactions are hermitian. The operators in grey arise only at one loop in weakly-coupled renormalisable UV completions of the SM [29].

is quadratic in the dimension-six couplings, can dominate the running of dimension-eight Wilson coefficients even if the latter are equally large. As such, the computation of this piece of the running is especially appealing.

Moreover, non-renormalisation theorems $29,40,42]$ have not been yet established for the mixing triggered by pairs of dimension-six operators. Consequently, for now the zeros in $\gamma^{\prime}$ can be only obtained upon explicit calculation.

We therefore focus on this part of the dimension-eight running in what follows. Likewise, and as a first attack to the problem, we will concentrate on the bosonic sector of the theory. The advantage of this is that bosonic operators are not renormalised by field-redefining away redundant operators involving fermions (the opposite is not true). Besides, we consider loops involving only dimension-six operators that can arise at tree level in weakly-coupled UV completions of the SM. These can be found in Refs. [43 47]. 


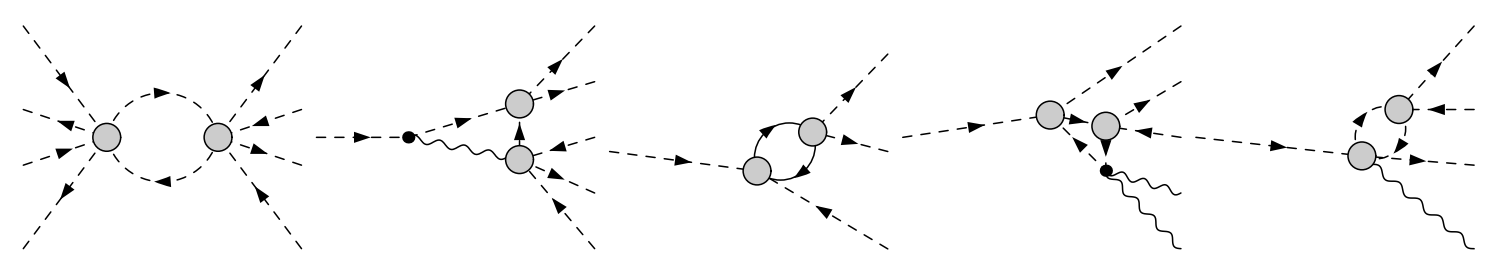

Figure 1: Example diagrams for the renormalisation of operators in classes $\phi^{8}$ (first), $\phi^{6} D^{2}$ (second), $\phi^{4} D^{2}$ (third), $X^{2} \phi^{4}$ (fourth) and $X \phi^{4} D^{2}$ (fifth). The gray blobs represent dimension-six interactions.

Thus, our starting Lagrangian is:

$$
\begin{aligned}
\mathcal{L}_{\mathrm{UV}} & =\mathcal{L}_{\mathrm{SM}}+\frac{1}{\Lambda^{2}}\left\{c_{\phi}\left(\phi^{\dagger} \phi\right)^{3}+c_{\phi \square}\left(\phi^{\dagger} \phi\right) \square\left(\phi^{\dagger} \phi\right)+c_{\phi D}\left(\phi^{\dagger} D^{\mu} \phi\right)^{*}\left(\phi^{\dagger} D_{\mu} \phi\right)\right. \\
& +c_{\phi \psi_{L}}^{(1)}\left(\phi^{\dagger} i \overleftrightarrow{D_{\mu}} \phi\right)\left(\overline{\psi_{L}} \gamma^{\mu} \psi_{L}\right)+c_{\phi \psi_{L}}^{(3)}\left(\phi^{\dagger} i \overleftrightarrow{D^{I}}{ }_{\mu}^{I} \phi\right)\left(\overline{\psi_{L}} \gamma^{\mu} \sigma^{I} \psi_{L}\right)+c_{\phi \psi_{R}}\left(\phi^{\dagger} i \overleftrightarrow{D_{\mu}} \phi\right)\left(\overline{\psi_{R}} \gamma^{\mu} \psi_{R}\right) \\
& \left.+\left[c_{\phi u d}\left(\widetilde{\phi} i D_{\mu} \phi\right)\left(\overline{u_{R}} \gamma^{\mu} d_{R}\right)+c_{\psi_{R} \phi}\left(\phi^{\dagger} \phi\right) \overline{\psi_{L}} \widetilde{\phi} \psi_{R}+\text { h.c. }\right]\right\}
\end{aligned}
$$

with $\psi_{R}=u_{R}, d_{R}, e_{R}$ and $\psi_{L}=q_{L}, l_{L}$. Restricting to the bosonic sector of the SMEFT, only dimension-eight operators involving Higgses can be renormalised at one loop from the Lagrangian above. For clarity, we reproduce them in Table 1 following the notation of Ref. [17].

\section{Computation}

We use the background field method and work in the Feynman gauge in dimensional regularisation with space-time dimension $\mathrm{d}=4-2 \epsilon$. We compute the one-loop divergences generated by $\mathcal{L}_{\text {UV }}$ using dedicated routines that rely on FeynRules [48, FeynArts 49$]$ and FormCalc [50]. Most of the calculations have been cross-checked using Match-Maker [51]. All amplitudes of the kind $X^{3} \phi^{2}$ and $X^{2} \phi^{2} D^{2}$ are finite, hence operators in these classes do not renormalise within our theory.

The bosonic one-loop divergent Lagrangian, involving Higgses, can be written as:

$$
16 \pi^{2} \epsilon \mathcal{L}_{\mathrm{DIV}}=\tilde{K}_{\phi}\left(D_{\mu} \phi\right)^{\dagger}\left(D^{\mu} \phi\right)-\tilde{\mu}^{2}|\phi|^{2}-\tilde{\lambda}|\phi|^{4}+\tilde{c}_{i}^{(6)} \frac{\mathcal{O}_{i}^{(6)}}{\Lambda^{2}}+\tilde{c}_{j}^{(8)} \frac{\mathcal{O}_{j}^{(8)}}{\Lambda^{4}}
$$

where $i$ and $j$ run over elements in the Green bases of operators of dimension-six and dimension-eight, respectively. The former extends the Warsaw basis with the interactions given in Table 2. The bosonic Higgs operators expanding the dimension-eight Green basis and which are redundant in the basis of Table 1 are shown in Table 3 . To the best of our knowledge, this last result is completely new.

We are interested in the unknown $\mathcal{O}\left(E^{4} / \Lambda^{4}\right)$ piece of the renormalisation of bosonic operators. As such, we only provide this new contribution to the aforementioned divergences. The only exception are the Higgs kinetic term and the dimension-six redundant operators, for which we also compute $E^{2} / \Lambda^{2}$ corrections, as these generate $E^{4} / \Lambda^{4}$ terms when moving to the physical basis by means of field redefinitions. Note also that, since we are dealing with only bosonic operators, we omit flavour indices. Flavourful couplings are written in matrix form (keeping the correct order in the matrix multiplication) and a trace over indices is implicit. We also use the shorthand notation for matrices $A^{2} \equiv \operatorname{Tr} A^{\dagger} A$, where $A$ is an arbitrary flavour matrix. 


\begin{tabular}{cclcl}
\hline & Operator & Notation & Operator & Notation \\
$\phi^{2} D^{4}$ & $\left(D_{\mu} D^{\mu} \phi^{\dagger}\right)\left(D_{\nu} D^{\nu} \phi\right)$ & $\mathcal{O}_{D \phi}$ & & \\
\hline$\phi^{4} D^{2}$ & $\left(\phi^{\dagger} \phi\right)\left(D_{\mu} \phi\right)^{\dagger}\left(D^{\mu} \phi\right)$ & $\mathcal{O}_{\phi D}^{\prime}$ & $\left(\phi^{\dagger} \phi\right) D^{\mu}\left(\phi^{\dagger} \mathrm{i} \overleftrightarrow{D}_{\mu} \phi\right)$ & $\mathcal{O}_{\phi D}^{\prime \prime}$ \\
\hline $\boldsymbol{X} \phi^{2} D^{2}$ & $D_{\nu} W^{I \mu \nu}\left(\phi^{\dagger} \mathrm{i} \overleftrightarrow{D}_{\mu}^{I} \phi\right)$ & $\mathcal{O}_{W D \phi}$ & $\partial_{\nu} B^{\mu \nu}\left(\phi^{\dagger} \mathrm{i} \overleftrightarrow{D}_{\mu} \phi\right)$ & $\mathcal{O}_{B D \phi}$ \\
\hline
\end{tabular}

Table 2: Independent dimension-six bosonic operators involving the Higgs which are redundant with respect to the Warsaw basis. We adopt the notation of Ref. [52].

\begin{tabular}{|c|c|c|c|c|}
\hline $\begin{array}{l}\stackrel{0}{a} \\
\text { â } \\
\text { Q }\end{array}$ & $\begin{array}{c}\text { Operator } \\
D^{2} \phi^{\dagger} D_{\mu} D_{\nu} D^{\mu} D^{\nu} \phi\end{array}$ & $\begin{array}{l}\text { Notation } \\
\mathcal{O}_{\phi^{2}}\end{array}$ & Operator & Notation \\
\hline$\stackrel{\vec{A}}{\vec{F}^{\prime}}$ & $\begin{array}{c}D_{\mu} \phi^{\dagger} D^{\mu} \phi\left(\phi^{\dagger} D^{2} \phi+\text { h.c. }\right) \\
\left(D_{\mu} \phi^{\dagger} \phi\right)\left(D^{2} \phi^{\dagger} D_{\mu} \phi\right)+\text { h.c. } \\
\left(D^{2} \phi^{\dagger} \phi\right)\left(D^{2} \phi^{\dagger} \phi\right)+\text { h.c. } \\
\quad\left(D^{2} \phi^{\dagger} D^{2} \phi\right)\left(\phi^{\dagger} \phi\right) \\
\left(D_{\mu} \phi^{\dagger} \phi\right)\left(D^{\mu} \phi^{\dagger} D^{2} \phi\right)+\text { h.c. }\end{array}$ & $\begin{array}{l}\mathcal{O}_{\phi^{4}}^{(4)} \\
\mathcal{O}_{\phi^{4}}^{(6)} \\
\mathcal{O}_{\phi^{4}}^{(8)} \\
\mathcal{O}_{\phi^{4}}^{(10)} \\
\mathcal{O}_{\phi^{4}}^{(12)}\end{array}$ & $\begin{array}{c}D_{\mu} \phi^{\dagger} D^{\mu} \phi\left(\phi^{\dagger} \mathrm{i} D^{2} \phi+\text { h.c. }\right) \\
\left(D_{\mu} \phi^{\dagger} \phi\right)\left(D^{2} \phi^{\dagger} \mathrm{i} D_{\mu} \phi\right)+\text { h.c. } \\
\left(D^{2} \phi^{\dagger} \phi\right)\left(\mathrm{i} D^{2} \phi^{\dagger} \phi\right)+\text { h.c. } \\
\quad\left(\phi^{\dagger} D^{2} \phi\right)\left(D^{2} \phi^{\dagger} \phi\right) \\
\left(D_{\mu} \phi^{\dagger} \phi\right)\left(D^{\mu} \phi^{\dagger} \mathrm{i} D^{2} \phi\right)+\text { h.c. }\end{array}$ & $\begin{array}{l}\mathcal{O}_{\phi^{4}}^{(5)} \\
\mathcal{O}_{\phi^{4}}^{(7)} \\
\mathcal{O}_{\phi^{4}}^{(9)} \\
\mathcal{O}_{\phi^{4}}^{(11)} \\
\mathcal{O}_{\phi^{4}}^{(13)}\end{array}$ \\
\hline $\begin{array}{l}0 \\
\hat{0} \\
0\end{array}$ & $\left(\phi^{\dagger} \phi\right)^{2}\left(\phi^{\dagger} D^{2} \phi+\right.$ h.c. $)$ & $\mathcal{O}_{\phi^{6}}^{(3)}$ & $\left(\phi^{\dagger} \phi\right)^{2} D_{\mu}\left(\phi^{\dagger} \mathrm{i} \overleftrightarrow{D}^{\mu} \phi\right)$ & $\mathcal{O}_{\phi^{6}}^{(4)}$ \\
\hline 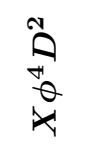 & $\begin{array}{c}\left(\phi^{\dagger} \phi\right) D_{\nu} W^{I \mu \nu}\left(D_{\mu} \phi^{\dagger} \sigma^{I} \phi+\text { h.c. }\right) \\
\epsilon^{I J K}\left(D_{\mu} \phi^{\dagger} \sigma^{I} \phi\right)\left(\phi^{\dagger} \sigma^{J} D_{\nu} \phi\right) W^{K \mu \nu}\end{array}$ & $\begin{array}{l}\mathcal{O}_{W \phi^{4} D^{2}}^{(5)} \\
\mathcal{O}_{W \phi^{4} D^{2}}^{(7)}\end{array}$ & $\begin{array}{c}\left(\phi^{\dagger} \phi\right) D_{\nu} W^{I \mu \nu}\left(D_{\mu} \phi^{\dagger} \mathrm{i} \sigma^{I} \phi+\text { h.c. }\right) \\
\left(\phi^{\dagger} \phi\right) D_{\nu} B^{\mu \nu}\left(D_{\mu} \phi^{\dagger} \mathrm{i} \phi+\text { h.c. }\right)\end{array}$ & $\begin{array}{l}\mathcal{O}_{W \phi^{4} D^{2}}^{(6)} \\
\mathcal{O}_{B \phi^{4} D^{2}}^{(3)}\end{array}$ \\
\hline
\end{tabular}

Table 3: Independent dimension-eight bosonic operators involving the Higgs which are redundant with respect to the basis of Ref. [17]. Redundant operators in the class $X^{2} \phi^{2} D^{2}$ are not shown. The addition of h.c. when needed implies that all operators are hermitian.

Thus, the divergences of the couplings of dimension $d \leq 4$ read:

$$
\begin{aligned}
\tilde{K}_{\phi} \supset & -\frac{1}{2}\left(c_{\phi D}+2 c_{\phi \square}\right) \frac{\mu^{2}}{\Lambda^{2}}, \\
\tilde{\lambda} & \supset-\frac{3}{2}\left(c_{\phi D}^{2}-4 c_{\phi D} c_{\phi \square}+8 c_{\phi \square}^{2}\right) \frac{\mu^{4}}{\Lambda^{4}} .
\end{aligned}
$$

We use the symbol $\supset$ to make explicit that corrections irrelevant for the computation of the $E^{4} / \Lambda^{4}$ terms are disregarded.

The $\tilde{c}^{(6)}$ couplings in Eq. 5 read:

$$
\begin{aligned}
& \tilde{c}_{\phi D} \supset c_{\phi D}\left(5 c_{\phi D}-8 c_{\phi \square}\right) \frac{\mu^{2}}{\Lambda^{2}}, \\
& \tilde{c}_{\phi \square} \supset \frac{1}{4}\left(c_{\phi D}^{2}+24 c_{\phi D} c_{\phi \square}-48 c_{\phi \square}^{2}\right) \frac{\mu^{2}}{\Lambda^{2}}, \\
& \tilde{c}_{\phi} \supset 6 c_{\phi}\left(3 c_{\phi D}-10 c_{\phi \square}\right) \frac{\mu^{2}}{\Lambda^{2}}+12 \lambda\left(c_{\phi D}^{2}-6 c_{\phi D} c_{\phi \square}+12 c_{\phi \square}^{2}\right) \frac{\mu^{2}}{\Lambda^{2}},
\end{aligned}
$$




$$
\begin{aligned}
\tilde{c}_{\phi D}^{\prime} & =\frac{1}{2}\left[\left(3 g_{2}^{2}-3 g_{1}^{2}-4 \lambda\right) c_{\phi D}+\left(8 \lambda-6 g_{2}^{2}\right) c_{\phi \square}\right] \\
& -\left[3\left(c_{u \phi} y^{u \dagger}+y^{u} c_{u \phi}^{\dagger}+y^{d} c_{d \phi}^{\dagger}+c_{d \phi} y^{d \dagger}\right)+y^{e} c_{e \phi}^{\dagger}+c_{e \phi} y^{e \dagger}\right] \\
& +12 c_{\phi q}^{(3)}\left(y^{u} y^{u \dagger}+y^{d} y^{d \dagger}\right)-6\left(c_{\phi u d} y^{d \dagger} y^{u}+c_{\phi u d}^{\dagger} y^{u \dagger} y^{d}\right)+4 c_{\phi l}^{(3)} y^{e} y^{e \dagger} \\
& -\left(c_{\phi D}^{2}+4 c_{\phi D} c_{\phi \square}-8 c_{\phi \square}^{2}\right) \frac{\mu^{2}}{\Lambda^{2}}, \\
\tilde{c}_{\phi D}^{\prime \prime} & =-\frac{i}{2}\left[3\left(c_{u \phi} y^{u \dagger}-y^{u} c_{u \phi}^{\dagger}+y^{d} c_{d \phi}^{\dagger}-c_{d \phi} y^{d \dagger}\right)+\left(y^{e} c_{e \phi}^{\dagger}-c_{e \phi} y^{e \dagger}\right)\right], \\
\tilde{c}_{B D \phi} & =-\frac{g_{1}}{6}\left[c_{\phi D}+c_{\phi \square}-4 c_{\phi e}-4 c_{\phi l}^{(1)}+8 c_{\phi u}-4 c_{\phi d}+4 c_{\phi q}^{(1)}\right], \\
\tilde{c}_{W D \phi} & =-\frac{g_{2}}{6}\left[c_{\phi \square}+4 c_{\phi l}^{(3)}+12 c_{\phi q}^{(3)}\right] ;
\end{aligned}
$$

while for the $\tilde{c}^{(8)}$ we get:

$$
\begin{aligned}
\tilde{c}_{\phi^{8}} & =\frac{3}{16}\left[336 c_{\phi}^{2}+\left(g_{1}^{4}+g_{2}^{4}+2 g_{1}^{2} g_{2}^{2}+160 \lambda^{2}\right) c_{\phi D}^{2}+2304 \lambda^{2} c_{\phi \square}^{2}\right. \\
& \left.+512 \lambda c_{\phi \square} c_{\phi}-1920 \lambda c_{\phi D} c_{\phi}-1152 \lambda^{2} c_{\phi \square} c_{\phi}\right] \\
& -\left[c_{e \phi} y^{e \dagger} c_{e \phi} y^{e \dagger}+c_{e \phi}^{\dagger} y^{e} c_{e \phi}^{\dagger} y^{e}+2 c_{e \phi} c_{e \phi}^{\dagger} y^{e} y^{e \dagger}+2 c_{e \phi}^{\dagger} c_{e \phi} y^{e \dagger} y^{e}\right] \\
& -3\left[c_{u \phi} y^{u \dagger} c_{u \phi} y^{u \dagger}+c_{u \phi}^{\dagger} y^{u} c_{u \phi}^{\dagger} y^{u}+2 c_{u \phi} c_{u \phi}^{\dagger} y^{u} y^{u \dagger}+2 c_{u \phi}^{\dagger} c_{u \phi} y^{u \dagger} y^{u}\right] \\
& -3\left[c_{d \phi} y^{d \dagger} c_{d \phi} y^{d \dagger}+c_{d \phi}^{\dagger} y^{d} c_{d \phi}^{\dagger} y^{d}+2 c_{d \phi} c_{d \phi}^{\dagger} y^{d} y^{d \dagger}+2 c_{d \phi}^{\dagger} c_{d \phi} y^{d \dagger} y^{d}\right] \\
\tilde{c}_{\phi^{4}}^{(1)} & =\frac{1}{6}\left[11 c_{\phi D}^{2}-32 c_{\phi D} c_{\phi \square}+16 c_{\phi \square}^{2}+24 c_{\phi u d}^{2}-24 c_{\phi d}^{2}-8 c_{\phi e}^{2}-16\left(c_{\phi l}^{(1)}\right)^{2}\right. \\
& \left.+16\left(c_{\phi l}^{(3)}\right)^{2}-48\left(c_{\phi q}^{(1)}\right)^{2}+48\left(c_{\phi q}^{(3)}\right)^{2}-24 c_{\phi u}^{2}\right], \\
\tilde{c}_{\phi^{4}}^{(2)} & =\frac{1}{6}\left[5 c_{\phi D}^{2}+16 c_{\phi D} c_{\phi \square}+16 c_{\phi \square}^{2}+24 c_{\phi d}^{2}+8 c_{\phi e}^{2}+16\left(c_{\phi l}^{(1)}\right)^{2}+16\left(c_{\phi l}^{(3)}\right)^{2}+48\left(c_{\phi q}^{(1)}\right)^{2}\right. \\
& \left.+48\left(c_{\phi q}^{(3)}\right)^{2}+24 c_{\phi u}^{2}\right] \\
\tilde{c}_{\phi^{4}}^{(3)} & =\frac{1}{6}\left[-7 c_{\phi D}^{2}+16 c_{\phi D} c_{\phi \square}+40 c_{\phi \square}^{2}-32\left(c_{\phi l}^{(3)}\right)^{2}-96\left(c_{\phi q}^{(3)}\right)^{2}-24 c_{\phi u d}^{2}\right] \\
\tilde{c}_{\phi^{4}}^{(4)} & =\frac{1}{2}\left[-c_{\phi D}^{2}-2 c_{\phi D} c_{\phi \square}+24 c_{\phi \square}^{2}\right] \\
\tilde{c}_{\phi^{4}}^{(6)} & =c_{\phi D}\left(c_{\phi D}-c_{\phi \square}\right), \\
\tilde{c}_{\phi^{4}}^{(8)} & =\frac{1}{8}\left[c_{\phi D}^{2}-8 c_{\phi D} c_{\phi \square}+32 c_{\phi \square}^{2}\right] \\
\tilde{c}_{\phi^{4}}^{(10)} & =\frac{1}{2} c_{\phi D}^{2}, \\
\tilde{c}_{\phi^{4}}^{(11)} & =\frac{1}{4}\left[c_{\phi D}^{2}-8 c_{\phi D} c_{\phi \square}+32 c_{\phi \square}^{2}\right] \\
\tilde{c}_{\phi^{4}}^{(12)} & =\frac{1}{2} c_{\phi D}\left(c_{\phi D}+2 c_{\phi \square}\right) \\
&
\end{aligned}
$$




$$
\begin{aligned}
& \tilde{c}_{\phi^{6}}^{(1)}=\frac{1}{4}\left[24 c_{\phi}\left(c_{\phi D}+8 c_{\phi \square}\right)-2 c_{\phi D} c_{\phi \square}\left(9 g_{1}^{2}+3 g_{2}^{2}-32 \lambda\right)\right. \\
&\left.+c_{\phi D}^{2}\left(-3 g_{1}^{2}-9 g_{2}^{2}+34 \lambda\right)-4 c_{\phi \square}^{2}\left(9 g_{1}^{2}+15 g_{2}^{2}+112 \lambda\right)\right] \\
&-3\left(c_{d \phi}^{2}+c_{u \phi}^{2}\right)-c_{e \phi}^{2}-\left(3 c_{\phi l}^{(1)}+5 c_{\phi l}^{(3)}\right)\left[y^{e} c_{e \phi}^{\dagger}+c_{e \phi} y^{e \dagger}\right] \\
&+9 c_{\phi q}^{(1)}\left[y^{u} c_{u \phi}^{\dagger}+c_{u \phi} y^{u \dagger}-y^{d} c_{d \phi}^{\dagger}-c_{d \phi} y^{d \dagger}\right]-15 c_{\phi q}^{(3)}\left[y^{u} c_{u \phi}^{\dagger}+c_{u \phi} y^{u \dagger}+y^{d} c_{d \phi}^{\dagger}+c_{d \phi} y^{d \dagger}\right] \\
&-3\left[3\left(c_{\phi u} c_{u \phi}^{\dagger} y^{u}+c_{u \phi} c_{\phi u} y^{u \dagger}-c_{d \phi} c_{\phi d} y^{d \dagger}-c_{\phi d} c_{d \phi}^{\dagger} y^{d}\right)-c_{e \phi} c_{\phi e} y^{e \dagger}-c_{\phi e} c_{e \phi}^{\dagger} y^{e}\right] \\
&-9\left[2\left(c_{\phi q}^{(1)}-c_{\phi q}^{(3)}\right) y^{u} c_{\phi u} y^{u \dagger}+2\left(c_{\phi q}^{(1)}+c_{\phi q}^{(3)}\right) y^{d} c_{\phi d} y^{d \dagger}\right]+9\left(c_{\phi q}^{(1)} c_{\phi q}^{(3)}+c_{\phi q}^{(3)} c_{\phi q}^{(1)}\right)\left[-y^{u} y^{u \dagger}+y^{d} y^{d \dagger}\right] \\
&+15 c_{\phi q}^{(3)}\left[y^{u} y^{u \dagger}+y^{d} y^{d \dagger}\right] c_{\phi q}^{(3)}+9 c_{\phi q}^{(1)}\left[y^{u} y^{u \dagger}+y^{d} y^{d \dagger}\right] c_{\phi q}^{(1)} \\
&-3\left[2\left(c_{\phi l}^{(1)}+c_{\phi l}^{(3)}\right) y^{e} c_{\phi e} y^{e \dagger}-\left(c_{\phi l}^{(1)} c_{\phi l}^{(3)}+c_{\phi l}^{(3)} c_{\phi l}^{(1)}\right) y^{e} y^{e \dagger}\right]+5 c_{\phi l}^{(3)} y^{e} y^{e \dagger} c_{\phi l}^{(3)}+3 c_{\phi l}^{(1)} y^{e} y^{e \dagger} c_{\phi l}^{(1)} \\
&+\frac{3}{2}\left[c_{\phi u d} c_{\phi u d}^{\dagger} y^{u \dagger} y^{u}+c_{\phi u d}^{\dagger} c_{\phi u d} y^{d \dagger} y^{d}\right]+3\left[3\left(c_{\phi u} y^{u \dagger} y^{u} c_{\phi u}+c_{\phi d} y^{d \dagger} y^{d} c_{\phi d}\right)+c_{\phi e} y^{e \dagger} y^{e} c_{\phi e}\right] \\
&-6 c_{\phi q}^{(3)}\left[y^{u} c_{\phi u d} y^{d \dagger}+y^{d} c_{\phi u d}^{\dagger} y^{u \dagger}\right]+3\left[c_{\phi u d} c_{d \phi}^{\dagger} y^{u}+c_{d \phi} c_{\phi u d}^{\dagger} y^{u \dagger}+c_{u \phi} c_{\phi u d} y^{d \dagger}+y^{d} c_{\phi u d}^{\dagger} c_{u \phi}^{\dagger}\right] \\
& \tilde{c}_{\phi^{6}}^{(2)}=\frac{1}{8}\left[72 c_{\phi} c_{\phi D}-24 c_{\phi \square}\left(c_{\phi D}+2 c_{\phi \square}\right) g_{1}^{2}-3 c_{\phi D}\left(c_{\phi D}+16 c_{\phi \square}\right) g_{2}^{2}\right. \\
&(27)
\end{aligned}
$$$$
\left.+32 c_{\phi D}\left(3 c_{\phi D}-8 c_{\phi \square}\right) \lambda\right]-3\left[c_{\phi u d} c_{d \phi}^{\dagger} y^{u}+c_{d \phi} c_{\phi u d}^{\dagger} y^{u \dagger}+y^{d} c_{\phi u d}^{\dagger} c_{u \phi}^{\dagger}+c_{u \phi} c_{\phi u d} y^{d \dagger}\right]
$$$$
-2\left[c_{\phi l}^{(1)} y^{e} c_{e \phi}^{\dagger}+c_{e \phi} y^{e \dagger} c_{\phi l}^{(1)}-3\left(c_{\phi q}^{(1)} y^{u} c_{u \phi}^{\dagger}+c_{u \phi} y^{u \dagger} c_{\phi q}^{(1)}-c_{\phi q}^{(1)} y^{d} c_{d \phi}^{\dagger}-c_{d \phi} y^{d \dagger} c_{\phi q}^{(1)}\right)\right]
$$$$
+2\left[c_{\phi e} c_{e \phi}^{\dagger} y^{e}+c_{e \phi} c_{\phi e} y^{e \dagger}-3\left(c_{\phi u} c_{u \phi}^{\dagger} y^{u}+c_{u \phi} c_{\phi u} y^{u \dagger}-c_{\phi d} c_{d \phi}^{\dagger} y^{d}-c_{d \phi} c_{\phi d} y^{d \dagger}\right)\right]
$$$$
-4\left[c_{\phi l}^{(1)}+c_{\phi l}^{(3)}\right] y^{e} c_{\phi e} y^{e \dagger}-12\left[\left(c_{\phi q}^{(1)}-c_{\phi q}^{(3)}\right) y^{u} c_{\phi u} y^{u \dagger}+\left(c_{\phi q}^{(1)}+c_{\phi q}^{(3)}\right) y^{d} c_{\phi d} y^{d \dagger}\right]
$$$$
+2\left[c_{\phi l}^{(1)} c_{\phi l}^{(3)}+c_{\phi l}^{(3)} c_{\phi l}^{(1)}+c_{\phi l}^{(1)} c_{\phi l}^{(1)}\right] y^{e} y^{e \dagger}+2 c_{\phi e} c_{\phi e} y^{e \dagger} y^{e}-6\left[c_{\phi q}^{(1)} c_{\phi q}^{(3)}+c_{\phi q}^{(3)} c_{\phi q}^{(1)}-c_{\phi q}^{(1)} c_{\phi q}^{(1)}\right] y^{u} y^{u \dagger}
$$$$
+6 c_{\phi u} c_{\phi u} y^{u \dagger} y^{u}+6\left[c_{\phi q}^{(1)} c_{\phi q}^{(3)}+c_{\phi q}^{(3)} c_{\phi q}^{(1)}+c_{\phi q}^{(1)} c_{\phi q}^{(1)}\right] y^{d} y^{d \dagger}+6 c_{\phi d} c_{\phi d} y^{d \dagger} y^{d}
$$$$
-\frac{3}{2}\left[c_{\phi u d} c_{\phi u d}^{\dagger} y^{u \dagger} y^{u}+c_{\phi u d}^{\dagger} c_{\phi u d} y^{d \dagger} y^{d}\right]+6 c_{\phi q}^{(3)}\left[y^{d} c_{\phi u d}^{\dagger} y^{u \dagger}+y^{u} c_{\phi u d} y^{d \dagger}\right],
$$$$
\tilde{c}_{\phi^{6}}^{(3)}=\frac{1}{16}\left[432 c_{\phi} c_{\phi \square}+\left(448 \lambda-12 g_{1}^{2}-12 g_{2}^{2}\right) c_{\phi D} c_{\phi \square}-48 c_{\phi} c_{\phi D}\right.
$$$$
\left.-8\left(3 g_{1}^{2}+3 g_{2}^{2}+128 \lambda\right) c_{\phi \square}^{2}-\left(3 g_{2}^{2}+12 \lambda\right) c_{\phi D}^{2}\right]
$$$$
-c_{e \phi}^{2}-3\left(c_{d \phi}^{2}+c_{u \phi}^{2}\right)-\frac{1}{2}\left[\left(c_{\phi l}^{(1)}+c_{\phi l}^{(3)}\right)\left(y^{e} c_{e \phi}^{\dagger}+c_{e \phi} y^{e \dagger}\right)-c_{\phi e} c_{e \phi}^{\dagger} y^{e}-c_{e \phi} c_{\phi e} y^{e \dagger}\right]
$$$$
-\left[c_{\phi l}^{(1)}+c_{\phi l}^{(3)}\right] y^{e} c_{\phi e} y^{e \dagger}+\frac{1}{2}\left[c_{\phi l}^{(1)} c_{\phi l}^{(3)}+c_{\phi l}^{(1)} c_{\phi l}^{(1)}+c_{\phi l}^{(3)} c_{\phi l}^{(1)}+c_{\phi l}^{(3)} c_{\phi l}^{(3)}\right] y^{e} y^{e \dagger}+\frac{1}{2} c_{\phi e} c_{\phi e} y^{e \dagger} y^{e}
$$$$
-\frac{3}{2}\left[c_{\phi q}^{(1)}+c_{\phi q}^{(3)}\right]\left(y^{d} c_{d \phi}^{\dagger}+c_{d \phi} y^{d \dagger}\right)+\frac{3}{2}\left[c_{\phi d} c_{d \phi}^{\dagger} y^{d}+c_{d \phi} c_{\phi d} y^{d \dagger}\right]-3\left[c_{\phi q}^{(1)}+c_{\phi q}^{(3)}\right] y^{d} c_{\phi d} y^{d \dagger}
$$ 


$$
\begin{aligned}
& +\frac{3}{2}\left[c_{\phi q}^{(1)} c_{\phi q}^{(3)}+c_{\phi q}^{(1)} c_{\phi q}^{(1)}+c_{\phi q}^{(3)} c_{\phi q}^{(1)}+c_{\phi q}^{(3)} c_{\phi q}^{(3)}\right] y^{d} y^{d \dagger}+\frac{3}{2} c_{\phi d} c_{\phi d} y^{d \dagger} y^{d} \\
& -\frac{3}{2}\left[\left(-c_{\phi q}^{(1)}+c_{\phi q}^{(3)}\right)\left(y^{u} c_{u \phi}^{\dagger}+c_{u \phi} y^{u \dagger}\right)+c_{\phi u} c_{u \phi}^{\dagger} y^{u}+c_{u \phi} c_{\phi u} y^{u \dagger}\right]-3\left[c_{\phi q}^{(1)}-c_{\phi q}^{(3)}\right] y^{u} c_{\phi u} y^{u \dagger} \\
& +\frac{3}{2}\left[-c_{\phi q}^{(1)} c_{\phi q}^{(3)}+c_{\phi q}^{(1)} c_{\phi q}^{(1)}-c_{\phi q}^{(3)} c_{\phi q}^{(1)}+c_{\phi q}^{(3)} c_{\phi q}^{(3)}\right] y^{u} y^{u \dagger}+\frac{3}{2} c_{\phi u} c_{\phi u} y^{u \dagger} y^{u}, \\
& \tilde{c}_{\phi^{6}}^{(4)}=-\frac{3 \mathrm{i}}{2}\left[c_{d \phi}^{\dagger}\left(c_{\phi q}^{(1)}+c_{\phi q}^{(3)}\right) y^{d}-c_{\phi d} c_{d \phi}^{\dagger} y^{d}+c_{d \phi} c_{\phi d} y^{d \dagger}-\left(c_{\phi q}^{(1)}+c_{\phi q}^{(3)}\right) c_{d \phi} y^{d \dagger}\right. \\
& \left.+c_{u \phi}^{\dagger}\left(c_{\phi q}^{(1)}-c_{\phi q}^{(3)}\right) y^{u}-c_{\phi u} c_{u \phi}^{\dagger} y^{u}+c_{u \phi} c_{\phi u} y^{u \dagger}-\left(c_{\phi q}^{(1)}-c_{\phi q}^{(3)}\right) c_{u \phi} y^{u \dagger}\right] \\
& -\frac{\mathrm{i}}{2}\left[c_{e \phi}^{\dagger}\left(c_{\phi l}^{(1)}+c_{\phi l}^{(3)}\right) y^{e}-c_{\phi e} c_{e \phi}^{\dagger} y^{e}+c_{e \phi} c_{\phi e} y^{e \dagger}-\left(c_{\phi l}^{(1)}+c_{\phi l}^{(3)}\right) c_{e \phi} y^{e \dagger}\right], \\
& \tilde{c}_{W^{2} \phi^{4}}^{(1)}=\frac{g_{2}^{2}}{12}\left[c_{\phi D}^{2}-3 c_{\phi D} c_{\phi \square}-12\left(c_{\phi l}^{(3)}\right)^{2}-36\left(c_{\phi q}^{(3)}\right)^{2}+9 c_{\phi u d}^{2}\right], \\
& \tilde{c}_{W^{2} \phi^{4}}^{(3)}=\frac{g_{2}^{2}}{48}\left[c_{\phi D}^{2}-12 c_{\phi D} c_{\phi \square}-24 c_{\phi d}^{2}-8 c_{\phi e}^{2}-16\left(c_{\phi l}^{(1)}\right)^{2}-48\left(c_{\phi q}^{(1)}\right)^{2}-24 c_{\phi u}^{2}+12 c_{\phi u d}^{2}\right], \\
& \tilde{c}_{W B \phi^{4}}^{(1)}=\frac{g_{1} g_{2}}{24}\left[c_{\phi D}^{2}-12 c_{\phi D} c_{\phi \square}-8 c_{\phi e}^{2}-16\left(c_{\phi l}^{(1)}\right)^{2}-48\left(c_{\phi q}^{(1)}\right)^{2}-24 c_{\phi u}^{2}+12 c_{\phi u d}-24 c_{\phi d}^{2}\right], \\
& \tilde{c}_{B^{2} \phi^{4}}^{(1)}=-\frac{g_{1}^{2}}{48}\left[3 c_{\phi D}^{2}+24 c_{\phi d}^{2}+8 c_{\phi e}^{2}+16\left(c_{\phi l}^{(1)}\right)^{2}-48\left(c_{\phi l}^{(3)}\right)^{2}+48\left(c_{\phi q}^{(1)}\right)^{2}\right. \\
& \left.-144\left(c_{\phi q}^{(3)}\right)^{2}+24 c_{\phi u}^{2}+24 c_{\phi u d}^{2}\right] \\
& \tilde{c}_{W \phi^{4} D^{2}}^{(1)}=\frac{g_{2}}{6}\left[5 c_{\phi D}^{2}-24 c_{\phi D} c_{\phi \square}-8 c_{\phi e}^{2}-16\left(c_{\phi l}^{(1)}\right)^{2}-48\left(c_{\phi l}^{(3)}\right)^{2}-48\left(c_{\phi q}^{(1)}\right)^{2}\right. \\
& \left.-144\left(c_{\phi q}^{(3)}\right)^{2}-24 c_{\phi u}^{2}+48 c_{\phi u d}^{2}-24 c_{\phi d}^{2}\right] \text {, } \\
& \tilde{c}_{B \phi^{4} D^{2}}^{(1)}=-\frac{g_{1}}{6}\left[24 c_{\phi d}^{2}+3 c_{\phi D}^{2}+8\left(c_{\phi e}^{2}+2\left(c_{\phi l}^{(1)}\right)^{2}-6\left(c_{\phi l}^{(3)}\right)^{2}+6\left(c_{\phi q}^{(1)}\right)^{2}-18\left(c_{\phi q}^{(3)}\right)^{2}+3 c_{\phi u}^{2}\right)+24 c_{\phi u d}^{2}\right] \text {, } \\
& \tilde{c}_{W \phi^{4} D^{2}}^{(6)}=\frac{g_{2}}{24}\left[5 c_{\phi D}^{2}-20 c_{\phi D} c_{\phi \square}+16\left(c_{\phi \square}^{2}-2\left(c_{\phi l}^{(3)}\right)^{2}-6\left(c_{\phi q}^{(3)}\right)^{2}\right)+48 c_{\phi u d}^{2}\right], \\
& \tilde{c}_{W \phi^{4} D^{2}}^{(7)}=\frac{g_{2}}{6}\left[24 c_{\phi d}^{2}-c_{\phi D}\left(c_{\phi D}-12 c_{\phi \square}\right)+8\left(c_{\phi e}^{2}+2\left(c_{\phi l}^{(1)}\right)^{2}+6\left(c_{\phi q}^{(1)}\right)^{2}+3 c_{\phi u}^{2}\right)-12 c_{\phi u d}^{2}\right], \\
& \tilde{c}_{B \phi^{4} D^{2}}^{(3)}=\frac{g_{1}}{24}\left[-3 c_{\phi D}^{2}+4 c_{\phi D} c_{\phi \square}+16\left(c_{\phi \square}^{2}+4\left(c_{\phi l}^{(3)}\right)^{2}+12\left(c_{\phi q}^{(3)}\right)^{2}\right)-24 c_{\phi u d}^{2}\right] .
\end{aligned}
$$

All other relevant counterterms $\left(\tilde{\mu}^{2}, \tilde{c}_{D \phi}, \tilde{c}_{\phi^{4}}^{(5,7,9,13)}\right.$, etc. $)$ vanish at the order of $E / \Lambda$ we are interested in.

\section{The structure of the renormalisation group equations}

The explicit form of the divergences in the Green basis, shown in the equations above, is of utmost importance, or else the computation of RGEs at higher orders or involving other light degrees of freedom could not be built on our results 53. Subsequently, though, one 
171

\begin{tabular}{c|cccccccc}
$\gamma_{\mathbf{c}_{\phi}^{8}}^{\prime}$ & $c_{\phi} c_{\phi D}$ & $c_{\phi \square}$ & $c_{\phi \psi_{L}}^{(1)}$ & $c_{\phi \psi_{L}}^{(3)}$ & $c_{\phi \psi_{R}}$ & $c_{\phi u d}$ & $c_{\psi_{R} \phi}$ \\
\hline$c_{\phi}$ & $\times$ & $\times$ & $\times$ & 0 & $\times$ & 0 & $\times$ & $\times$ \\
$c_{\phi D}$ & $\times$ & $\times$ & $\times$ & $\times$ & $\times$ & $\times$ & $\times$ \\
$c_{\phi}$ & & & $\times$ & 0 & $\times$ & 0 & $\times$ & $\times$ \\
$c_{\phi \psi_{L}}^{(1)}$ & & & & $\times$ & $\times$ & $\times$ & 0 & $\times$ \\
$c_{\phi \psi_{L}}^{(3)}$ & & & & & $\times$ & $\times$ & 0 & $\times$ \\
$c_{\phi \psi_{R}}$ & & & & & & $\times$ & 0 & $\times$ \\
$c_{\phi u d}$ & & & & & & & $\times$ & 0 \\
$c_{\psi_{R} \phi}$ & & & & & & & & $\times$
\end{tabular}

\begin{tabular}{c|cccccccc}
$\gamma_{\mathbf{c}_{\phi}^{(2)}}^{\prime}$ & \multicolumn{1}{c|}{$c_{\phi} c_{\phi D}$} & $c_{\phi \square}$ & $c_{\phi \psi_{L}}^{(1)} c_{\phi \psi_{L}}^{(3)} c_{\phi \psi_{R}}$ & $c_{\phi u d}$ & $c_{\psi_{R} \phi}$ \\
\hline$c_{\phi}$ & 0 & 0 & 0 & 0 & 0 & 0 & 0 & 0 \\
$c_{\phi D}$ & & $\times$ & $\times$ & 0 & 0 & 0 & 0 & 0 \\
$c_{\phi \square}$ & & & $\times$ & 0 & 0 & 0 & 0 & 0 \\
$c_{\phi \psi_{L}}^{(1)}$ & & & & $\times$ & 0 & 0 & 0 & 0 \\
$c_{\phi \psi_{L}}^{(3)}$ & & & & & $\times$ & 0 & 0 & 0 \\
$c_{\phi \psi_{R}}$ & & & & & & $\times$ & 0 & 0 \\
$c_{\phi u d}$ & & & & & & & 0 & 0 \\
$c_{\psi_{R} \phi}$ & & & & & & & & 0
\end{tabular}

173

\begin{tabular}{c|cccccccc}
$\gamma_{\mathbf{c}_{\phi}^{(1)}}^{\prime}$ & $c_{\phi} c_{\phi D}$ & $c_{\phi \square}$ & $c_{\phi \psi_{L}}^{(1)}$ & $c_{\phi \psi_{L}}^{(3)}$ & $c_{\phi \psi_{R}}$ & $c_{\phi u d}$ & $c_{\psi_{R} \phi}$ \\
\hline$c_{\phi}$ & 0 & $\times$ & $\times$ & 0 & 0 & 0 & 0 & 0 \\
$c_{\phi D}$ & & $\times$ & $\times$ & $\times$ & $\times$ & $\times$ & $\times$ & $\times$ \\
$c_{\phi \square}$ & & & $\times$ & 0 & $\times$ & 0 & $\times$ & $\times$ \\
$c_{\phi \psi_{L}}^{(1)}$ & & & & $\times$ & $\times$ & $\times$ & 0 & $\times$ \\
$c_{\phi \psi_{L}}^{(3)}$ & & & & & $\times$ & $\times$ & $\times$ & $\times$ \\
$c_{\phi \psi_{R}}$ & & & & & & $\times$ & 0 & $\times$ \\
$c_{\phi u d}$ & & & & & & & $\times$ & $\times$ \\
$c_{\psi_{R} \phi}$ & & & & & & & & $\times$
\end{tabular}
Appendix A We find:

\begin{tabular}{c|cccccccc}
$\gamma_{\mathbf{c}_{\phi \mathbf{4}}^{(\mathbf{1})}}^{\prime}$ & $c_{\phi} c_{\phi D}$ & $c_{\phi \square}$ & $c_{\phi \psi_{L}}^{(1)} c_{\phi \psi_{L}}^{(3)} c_{\phi \psi_{R}}$ & $c_{\phi u d}$ & $c_{\psi_{R} \phi}$ \\
\hline$c_{\phi}$ & 0 & 0 & 0 & 0 & 0 & 0 & 0 & 0 \\
$c_{\phi D}$ & & $\times$ & $\times$ & 0 & 0 & 0 & 0 & 0 \\
$c_{\phi \square}$ & & & $\times$ & 0 & 0 & 0 & 0 & 0 \\
$c_{\phi \psi_{L}}^{(1)}$ & & & & $\times$ & 0 & 0 & 0 & 0 \\
$c_{\phi \psi_{L}}^{(3)}$ & & & & & $\times$ & 0 & 0 & 0 \\
$c_{\phi \psi_{R}}$ & & & & & & $\times$ & 0 & 0 \\
$c_{\phi u d}$ & & & & & & & $\times$ & 0 \\
$c_{\psi_{R} \phi}$ & & & & & & & & 0
\end{tabular}

\begin{tabular}{c|ccccccccc}
$\gamma_{\mathbf{c}_{\phi}^{(3)}}^{\prime}$ & \multicolumn{1}{|c|}{$c_{\phi} c_{\phi D}$} & $c_{\phi \square}$ & $c_{\phi \psi_{L}}^{(1)} c_{\phi \psi_{L}}^{(3)} c_{\phi \psi_{R}}$ & $c_{\phi u d}$ & $c_{\psi_{R} \phi}$ \\
\hline$c_{\phi}$ & 0 & 0 & 0 & 0 & 0 & 0 & 0 & 0 \\
$c_{\phi D}$ & & $\times$ & $\times$ & 0 & 0 & 0 & 0 & 0 \\
$c_{\phi \square}$ & & & $\times$ & 0 & 0 & 0 & 0 & 0 \\
$c_{\phi \psi_{L}}^{(1)}$ & & & & 0 & 0 & 0 & 0 & 0 \\
$c_{\phi \psi_{L}}^{(3)}$ & & & & & $\times$ & 0 & 0 & 0 \\
$c_{\phi \psi_{R}}$ & & & & & & 0 & 0 & 0 \\
$c_{\phi u d}$ & & & & & & & $\times$ & 0 \\
$c_{\psi_{R} \phi}$ & & & & & & & & 0
\end{tabular}

\begin{tabular}{c|cccccccc}
$\gamma_{\mathbf{c}_{\phi}^{(2)}}^{\prime(2)}$ & $c_{\phi} c_{\phi D}$ & $c_{\phi \square}$ & $c_{\phi \psi_{L}}^{(1)}$ & $c_{\phi \psi_{L}}^{(3)}$ & $c_{\phi \psi_{R}}$ & $c_{\phi u d}$ & $c_{\psi_{R} \phi}$ \\
\hline$c_{\phi}$ & 0 & $\times$ & 0 & 0 & 0 & 0 & 0 & 0 \\
$c_{\phi D}$ & & $\times$ & $\times$ & $\times$ & $\times$ & $\times$ & $\times$ & $\times$ \\
$c_{\phi \square}$ & & & $\times$ & 0 & 0 & 0 & 0 & 0 \\
$c_{\phi \psi_{L}}^{(1)}$ & & & & $\times$ & $\times$ & $\times$ & 0 & $\times$ \\
$c_{\phi \psi_{L}}^{(3)}$ & & & & & $\times$ & $\times$ & $\times$ & 0 \\
$c_{\phi \psi_{R}}$ & & & & & & $\times$ & 0 & $\times$ \\
$c_{\phi u d}$ & & & & & & & $\times$ & $\times$ \\
$c_{\psi_{R} \phi}$ & & & & & & & & $\times$
\end{tabular}

can reduce the redundant operators to the physical basis. We do that following the results

$$
16 \pi^{2} \mu \frac{d c_{i}^{(8)}}{d \mu}=-c_{i}^{(8)} \sum_{j} x_{j} n_{j} \frac{\partial}{\partial x_{j}} \frac{\tilde{c}_{i}^{(8)}}{c_{i}^{(8)}},
$$

with $x$ running over all couplings, renormalisable or not, and with $n$ representing the corresponding tree-level anomalous dimension, defined as the value required to keep the couplings dimensionless in $4-2 \epsilon$ dimensions. The minus sign results from requiring that counterterms cancel the divergences. The complete set of RGEs can be found in init ourselves to discussing the structure of the $\gamma^{\prime}$ matrices defined in Eq. (3). metric) matrix for each $c^{(8)}$ in which we represent with a $\times$ a non-vanishing entry, with 0 a trivial zero, for which all contributions in the Green basis vanish (for example in some cases there are no diagrams contributing to the corresponding amplitude), and with $\emptyset \mathrm{a}$ non-trivial zero, for which several non-vanishing contributions cancel in the physical basis. 


\begin{tabular}{|c|c|c|c|c|c|c|c|c|}
\hline$\gamma_{\mathbf{w}^{2} \phi^{4}}^{\prime(1)}$ & \multicolumn{8}{|c|}{$c_{\phi} c_{\phi D} c_{\phi \square} c_{\phi \psi_{L}}^{(1)} c_{\phi \psi_{L}}^{(3)} c_{\phi \psi_{R}} c_{\phi u d} c_{\psi_{R}}$} \\
\hline$c_{\phi}$ & 0 & 0 & 0 & 0 & 0 & 0 & 0 & 0 \\
\hline$c_{\phi D}$ & & $\times$ & $\emptyset$ & 0 & 0 & 0 & 0 & 0 \\
\hline$c_{\phi \square}$ & & & 0 & 0 & 0 & 0 & 0 & 0 \\
\hline$c_{\phi \psi_{L}}^{(1)}$ & & & & $x$ & 0 & 0 & 0 & 0 \\
\hline$c_{\phi \psi_{L}}^{(3)^{L}}$ & & & & & $\times$ & 0 & 0 & 0 \\
\hline$c_{\phi \psi_{R}}$ & & & & & & $\times$ & 0 & 0 \\
\hline$c_{\phi u d}$ & & & & & & & $\times$ & 0 \\
\hline$c_{\psi_{R} \phi}$ & & & & & & & & 0 \\
\hline
\end{tabular}

\begin{tabular}{c|cccccccc}
$\gamma_{\mathbf{c}_{\mathbf{W}^{2} \phi^{4}}^{(3)}}^{\prime}$ & \multicolumn{1}{c|}{$c_{\phi} c_{\phi D} c_{\phi \square}$} & $c_{\phi \psi_{L}}^{(1)}$ & $c_{\phi \psi_{L}}^{(3)}$ & $c_{\phi \psi_{R}}$ & $c_{\phi u d}$ & $c_{\psi_{R} \phi}$ \\
\hline$c_{\phi}$ & 0 & 0 & 0 & 0 & 0 & 0 & 0 & 0 \\
$c_{\phi D}$ & & $\emptyset$ & $\emptyset$ & 0 & 0 & 0 & 0 & 0 \\
$c_{\phi \square}$ & & & 0 & 0 & 0 & 0 & 0 & 0 \\
$c_{\phi \psi_{L}}^{(1)}$ & & & & $\emptyset$ & 0 & 0 & 0 & 0 \\
$c_{\phi \psi_{L}}^{(3)}$ & & & & & 0 & 0 & 0 & 0 \\
$c_{\phi \psi_{R}}$ & & & & & & $\emptyset$ & 0 & 0 \\
$c_{\phi u d}$ & & & & & & & $\emptyset$ & 0 \\
$c_{\psi_{R} \phi}$ & & & & & & & & 0
\end{tabular}

\begin{tabular}{|c|c|c|c|c|c|c|c|c|}
\hline$\gamma_{\mathbf{c}_{\mathrm{WB} \phi^{4}}^{(1)}}^{\prime}$ & \multicolumn{8}{|c|}{$c_{\phi} c_{\phi D} c_{\phi \square} c_{\phi \psi_{L}}^{(1)} c_{\phi \psi_{L}}^{(3)} c_{\phi \psi_{R}} c_{\phi u d} c_{\psi_{R} \phi}$} \\
\hline$c_{\phi}$ & 0 & 0 & $\overline{0}$ & 0 & 0 & 0 & $\overline{0}$ & 0 \\
\hline$c_{\phi D}$ & & $\emptyset$ & $l$ & 0 & 0 & 0 & 0 & 0 \\
\hline$c_{\phi \square}$ & & & 0 & 0 & 0 & 0 & 0 & 0 \\
\hline$c_{\phi \psi_{L}}^{(1)}$ & & & & $\emptyset$ & 0 & 0 & 0 & 0 \\
\hline$c_{\phi \psi_{L}}^{(3)^{2}}$ & & & & & 0 & 0 & 0 & 0 \\
\hline$c_{\phi \psi_{R}}$ & & & & & & $\emptyset$ & 0 & 0 \\
\hline$c_{\phi u d}$ & & & & & & & $\emptyset$ & 0 \\
\hline$c_{\psi_{R} \phi}$ & & & & & & & & 0 \\
\hline
\end{tabular}

\begin{tabular}{c|cccccccc}
$\gamma_{\mathbf{c}_{\mathbf{B}^{2} \phi^{4}}^{(1)}}^{\prime}$ & \multicolumn{1}{c|}{$c_{\phi} c_{\phi D} c_{\phi} \square$} & $c_{\phi \psi_{L}}^{(1)}$ & $c_{\phi \psi_{L}}^{(3)}$ & $c_{\phi \psi_{R}}$ & $c_{\phi u d}$ & $c_{\psi_{R} \phi}$ \\
\hline$c_{\phi}$ & 0 & 0 & 0 & 0 & 0 & 0 & 0 & 0 \\
$c_{\phi D}$ & & $\times$ & 0 & 0 & 0 & 0 & 0 & 0 \\
$c_{\phi \square}$ & & & 0 & 0 & 0 & 0 & 0 & 0 \\
$c_{\phi \psi_{L}}^{(1)}$ & & & & $\times$ & 0 & 0 & 0 & 0 \\
$c_{\phi \psi_{L}}^{(3)}$ & & & & & $\times$ & 0 & 0 & 0 \\
$c_{\phi \psi_{R}}$ & & & & & & $\times$ & 0 & 0 \\
$c_{\phi u d}$ & & & & & & & $\times$ & 0 \\
$c_{\psi_{R} \phi}$ & & & & & & & & 0
\end{tabular}

\begin{tabular}{|c|c|c|c|c|c|c|c|c|}
\hline$\gamma_{\mathbf{c}_{\mathbf{W}_{\phi} \mathbf{A}^{2} \mathbf{D}^{2}}^{\prime(1)}}^{\prime}$ & \multicolumn{8}{|c|}{$c_{\phi} c_{\phi D} c_{\phi \square} c_{\phi \psi_{L}}^{(1)} c_{\phi \psi_{L}}^{(3)} c_{\phi \psi_{R}} c_{\phi u d} c_{\psi_{R} \phi}$} \\
\hline$c_{\phi}$ & 0 & 0 & 0 & 0 & 0 & 0 & 0 & 0 \\
\hline$c_{\phi D}$ & & $x$ & $\emptyset$ & 0 & 0 & 0 & 0 & 0 \\
\hline$c_{\phi \square}$ & & & 0 & 0 & 0 & 0 & 0 & 0 \\
\hline$c_{\phi \psi_{L}}^{(1)}$ & & & & $\times$ & 0 & 0 & 0 & 0 \\
\hline$c_{\phi \psi_{L}}^{(3)^{L}}$ & & & & & $\times$ & 0 & 0 & 0 \\
\hline$c_{\phi \psi_{R}}$ & & & & & & $x$ & 0 & 0 \\
\hline$c_{\phi u d}$ & & & & & & & $\times$ & 0 \\
\hline$c_{\psi_{R} \phi}$ & & & & & & & & 0 \\
\hline
\end{tabular}

\begin{tabular}{|c|c|c|c|c|c|c|c|c|}
\hline$\gamma_{\mathbf{c}_{\mathbf{B} \phi^{4} \mathrm{D}^{2}}^{(1)}}^{\prime}$ & $c_{\phi}$ & $c_{\phi L}$ & & & $\begin{array}{l}\text { (3) } \\
\phi \psi_{L}\end{array}$ & $\phi \psi_{R}$ & ciud & $\psi_{R} \phi$ \\
\hline$c_{\phi}$ & 0 & 0 & $\overline{0}$ & 0 & 0 & 0 & 0 & 0 \\
\hline$c_{\phi D}$ & & $x$ & 0 & 0 & 0 & 0 & 0 & 0 \\
\hline$c_{\phi \square}$ & & & 0 & 0 & 0 & 0 & 0 & 0 \\
\hline$c_{\phi \psi_{L}}^{(1)}$ & & & & $x$ & 0 & 0 & 0 & 0 \\
\hline$c_{\phi \psi_{L}}^{(3)}$ & & & & & $x$ & 0 & 0 & 0 \\
\hline$c_{\phi \psi_{R}}$ & & & & & & $\times$ & 0 & 0 \\
\hline$c_{\phi u d}$ & & & & & & & $\times$ & 0 \\
\hline$c_{\psi_{R} \phi}$ & & & & & & & & 0 \\
\hline
\end{tabular}

All other $\gamma^{\prime}$ matrices vanish identically, with all their zeros being trivial.

Finally, in Table 4 we provide a different view on the global structure of the anomalous dimensions, by showing, for each pair of dimension-six interactions, the dimension-eight operators that get renormalised by them. Despite being not explicitly shown, contributions proportional to two fermionic operators involve only leptons or quarks, but not both. 


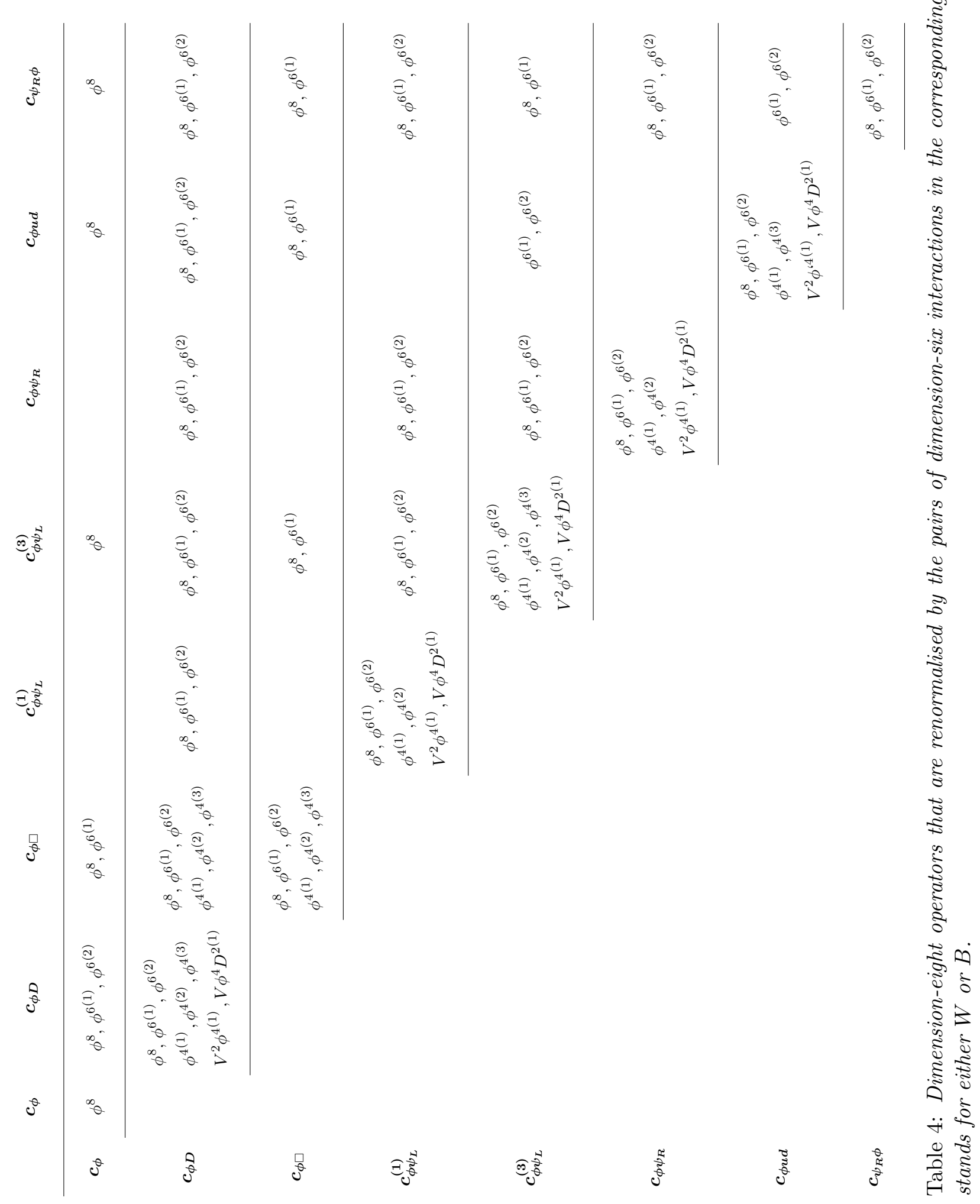




\section{Discussion and outlook}

We conclude this article highlighting several observations that can be made on the basis of the RGE matrices above:

1. All the dimension-eight operators that are renormalised can arise at tree level in UV completions of the SM [29]. The reason is simply that those operators that arise only at loop level involve two Higgs fields (see Table 1), unlike any one-loop diagram containing two insertions of the dimension-six terms. The same holds for dimension-six operators. Thus, we conclude that within the bosonic sector of the SMEFT, dimension-six tree-level operators do not mix into loop-level operators to order $E^{4} / \Lambda^{4}$. This extends previous findings at order $E^{2} / \Lambda^{2}$ 41].

2. Several of the $\gamma^{\prime}$ matrices above exhibit a number of zeros (denoted by 0 ) for which all contributions in the Green basis are vanishing (they can result simply from the absence of Feynman diagrams or from CP conservation reasons). For example, the first row in $\gamma_{c_{\phi^{4}}}^{\prime}$ reflects that there are no one-particle-irreducible diagrams with four Higgses involving the insertion of one six-Higgs operator and one four-Higgs interaction. Instead, those denoted by $\emptyset$ ensue from non-trivial cancellations between different counterterms in the Green basis which, on-shell, add to zero. For example, the (23) entry of $\gamma_{c_{W^{2} \phi^{4}}^{(1)}}^{\prime}$ vanishes because the terms proportional to $c_{\phi D} c_{\phi \square}$ in $\tilde{c}_{W^{2} \phi^{4}}^{(1)}$ and $\tilde{c}_{W \phi^{4} D^{2}}^{(7)}$ cancel in Eq. 73 . Zeros as this one might be understood on the basis of the helicity-amplitude formalism [54, 55].

3. Related to the previous point, we find the very surprising result that the PeskinTakeuchi parameters [56] $S$ and $U$ are not renormalised by tree-level dimension-six operators to order $v^{4} / \Lambda^{4}$. Indeed, these observables read [17,27]:

$$
\frac{1}{16 \pi} S=\frac{v^{2}}{\Lambda^{2}}\left[c_{\phi W B}+c_{W B \phi^{4}}^{(1)} \frac{v^{4}}{\Lambda^{4}}\right], \quad \frac{1}{16 \pi} U=\frac{v^{4}}{\Lambda^{4}} c_{W^{2} \phi^{4}}^{(3)}
$$

with $\mathcal{O}_{\phi W B}=\left(\phi^{\dagger} \sigma^{I} \phi\right) W_{\mu \nu}^{I} B^{\mu \nu}$. (Note that $U$ arises only at dimension eight.) What we find is that $c_{W B \phi^{4}}^{(1)}$ and $c_{W^{2} \phi^{4}}^{(3)}$ do not renormalise because the direct contribution cancels that from the redundant operator $\mathcal{O}_{W \phi^{4} D^{2}}^{(7)}$. This fact, together with the nonrenormalisation of $c_{\phi W B}$ found in Refs. [9], shows that both $S$ and $U$ are not triggered by dimension-six tree-level interactions at one loop.

4. The Wilson coefficients $c_{\phi^{4}}^{(1)}, c_{\phi^{4}}^{(2)}$ and $c_{\phi^{4}}^{(3)}$ are subject to positivity constraints 33 . In particular, $c_{\phi^{4}}^{(2)} \geq 0, c_{\phi^{4}}^{(1)}+c_{\phi^{4}}^{(2)} \geq 0$ and $c_{\phi^{4}}^{(1)}+c_{\phi^{4}}^{(2)}+c_{\phi^{4}}^{(3)} \geq 0$. These inequalities should reflect in the corresponding matrices.

To see how, let us first note that there exist well-behaved UV completions of the SM that induce, at tree level, the operators $c_{\phi}, c_{\phi D}$ and $c_{\phi}$ with arbitrary values; see Appendix C for a particular example. The values of the dimension-eight Wilson coefficients $c_{\phi^{4}}^{(1)}, c_{\phi^{4}}^{(2)}$ and $c_{\phi^{4}}^{(3)}$ at any energy $\mu<M$ triggered by double insertions of the dimensionsix operators scale differently with the model couplings than the tree-level contribution (which in general can not be avoided). In particular, within the model of Appendix C, we have $\sim \kappa^{4} / M^{4}$ versus $\sim \kappa^{2} / M^{2}$. This suggests that both contributions must satisfy the positivity bounds separately. Indeed, in the limit of scale-invariant dimension-six Wilson coefficients, we can check that:

$$
16 \pi^{2} c_{\phi^{4}}^{(2)}=\frac{1}{3}\left(5 c_{\phi D}^{2}+16 c_{\phi D} c_{\phi \square}+16 c_{\phi \square}^{2}\right) \log \frac{M}{\mu}>0,
$$




$$
\begin{aligned}
16 \pi^{2}\left[c_{\phi^{4}}^{(1)}+c_{\phi^{4}}^{(2)}\right] & =\frac{16}{3}\left(c_{\phi D}^{2}-c_{\phi D} c_{\phi \square}+2 c_{\phi \square}^{2}\right) \log \frac{M}{\mu}>0, \\
16 \pi^{2}\left[c_{\phi^{4}}^{(1)}+c_{\phi^{4}}^{(2)}+c_{\phi^{4}}^{(3)}\right] & =3\left(c_{\phi D}^{2}+8 c_{\phi \square}^{2}\right) \log \frac{M}{\mu}>0 ;
\end{aligned}
$$

for arbitrary values of $c_{\phi D}$ and $c_{\phi \square}$. (Fermionic Wilson coefficients do not modify these relations because they contribute as sums of modulus squared and therefore positively, as a result also of very fine cancellations between positive and negative terms in Eqs. $17,[19$.) Note that these inequalities hold non-trivially; for example $c_{\phi^{4}}^{(1)}$ is negative in a neighbourhood of its minimum. It should be possible to extend this kind of analysis to other operators (which do not renormalise within the assumptions we make in this work), thus providing interesting cross-checks of the anomalous dimensions (or new bounds on Wilson coefficients).

5. Among the non-vanishing entries in the different $\gamma^{\prime} \mathrm{s}$, we find values that depart significantly from the naive estimate of $\mathcal{O}(1)$. The most notable of these, not suppressed by gauge or $\lambda$ couplings, are the 126 in $\gamma_{\phi^{8}}^{\prime}$ and the 96 in $\gamma_{c_{\phi^{6}}}^{\prime(1)}$; see Appendix B. As we discuss below, these large numbers can have important low-energy implications.

6. Although we do not aim to exhaust all possible phenomenological implications of the running of the dimension-eight operators, we would like to stress that the $T$ parameter, defined by 17

$$
\alpha T=-\frac{1}{2} \frac{v^{2}}{\Lambda^{2}}\left[c_{\phi D}+c_{\phi^{6}}^{(2)} \frac{v^{2}}{\Lambda^{2}}\right]
$$

with $\alpha \sim 1 / 137$ being the fine-structure constant, receives contributions from the operator $\mathcal{O}_{\phi u d}$ only at order $v^{4} / \Lambda^{4}$ (because $c_{\phi D}$ is not renormalised by one insertion of $\mathcal{O}_{\phi u d}$; see Ref. [8]). Using bounds on $T$ from Ref. [57], and assuming that only $c_{\phi t b}$ is non-vanishing, we obtain $c_{\phi t b} \leq 5.9$ for $\Lambda=1 \mathrm{TeV}$. This constraint is competitive with the value $c_{\phi t b} \leq 5.3$ reported in Ref. [58].

7. We would also like to emphasize the importance of one-loop $v^{4} / \Lambda^{4}$ effects for the EW phase transition (EWPT) ensuing from modifications of the Higgs potential [16, 59 63. To this aim, let us assume that $c_{\phi}$ is the only non-vanishing Wilson coefficient in the UV, and let us neglect gauge and Yukawa couplings. The Higgs potential in the infrared is then provided by running $\mathcal{L}_{\mathrm{UV}}$ down to the EW scale. In the leading-logarithm approximation, this reads:

$$
V \sim \mu^{2}|\phi|^{2}+\lambda|\phi|^{4}+\frac{c_{\phi}}{\Lambda^{2}}\left(1-\frac{108}{16 \pi^{2}} \lambda \log \frac{\Lambda}{v}\right)|\phi|^{6}+\frac{126}{16 \pi^{2} \Lambda^{4}} \log \frac{\Lambda}{v} c_{\phi}^{2}|\phi|^{8},
$$

where $c_{\phi}$ is evaluated in the UV, and the renormalisable couplings are assumed scaleinvariant for simplicity. (The first logarithm can be read from Ref. [7].) In both $|\phi|^{6}$ and $|\phi|^{8}$ we have included only the dominant corrections.

Following Refs. [16, 64, we know that the EWPT is first order and strong as required by EW baryogenesis [65] provided that $500 \mathrm{GeV} \lesssim \Lambda / \sqrt{c_{\text {eff }}} \lesssim 750 \mathrm{GeV}$, where we have defined $c_{\text {eff }}=c_{\phi}+3 / 2 v^{2} / \Lambda^{2} c_{\phi^{8}}$. Fixing, as a matter of example, $\Lambda=1 \mathrm{TeV}$, it can be easily checked that this occurs for:

$$
1.7 \mathrm{TeV}^{-2} \lesssim c_{\phi} \lesssim 3.7 \mathrm{TeV}^{-2}
$$

if the running of $c_{\phi^{8}}$ is neglected, whereas if we account for it we obtain:

$$
1.5 \mathrm{TeV}^{-2} \lesssim c_{\phi} \lesssim 2.6 \mathrm{TeV}^{-2}
$$




\begin{tabular}{|c|c|c|c|c|c|c|c|c|c|c|c|}
\hline & $d_{5}$ & $d_{5}^{2}$ & $d_{6}$ & $d_{5}^{3}$ & $d_{5} \times d_{6}$ & $d_{7}$ & $d_{5}^{4}$ & $d_{5}^{2} \times d_{6}$ & $d_{6}^{2}$ & $d_{5} \times d_{7}$ & $d_{8}$ \\
\hline$d_{\leq 4}$ (bosonic) & & & $\begin{array}{l}\checkmark 7 \\
\end{array}$ & & & & & & This work & & $x$ \\
\hline$d_{\leq 4}$ (fermionic) & & & $\checkmark$ & & & & & & $\mathrm{x}$ & & $x$ \\
\hline$d_{5}$ & \begin{tabular}{ll|l}
$\checkmark$ & 66 & 68 \\
\end{tabular} & & & & $\checkmark 71$ & $\checkmark 71$ & & & & & \\
\hline$d_{6}$ (bosonic) & & $\checkmark 30$ & $\begin{array}{lll}\checkmark & 7 & 9 \\
\end{array}$ & & & & & $x$ & This work & $x$ & $x$ \\
\hline $\begin{array}{l}d_{6} \text { (fermionic) } \\
d_{7}\end{array}$ & & $\checkmark 30$ & $\begin{array}{llll}\checkmark & 7 & 9,69 \\
\end{array}$ & $\checkmark 71$ & $\checkmark 71$ & $\checkmark \quad 22,70$ & & $x$ & $x$ & $x$ & $x$ \\
\hline$d_{8}$ (bosonic) & & & & & & & $x$ & $x$ & This work & $x$ & $x$ \\
\hline$d_{8}$ (fermionic) & & & & & & & $x$ & $x$ & $x$ & $x$ & $x$ \\
\hline
\end{tabular}

Table 5: State of the art of SMEFT renormalisation. The rows represent the operators (defined by their dimension d) being renormalised, while the columns show the operators entering the loops. Note that there are no bosonic interactions at odd dimension. Blank entries vanish. A tick $\checkmark$ represents that the complete contribution is known. The $\checkmark$ indicates that only (but substantial) partial results have been already obtained. The $X$ indicates that nothing, or very little, is known. The contribution made in this paper is marked by

The $30 \%$ difference in the upper limit evidences the potential importance of both dimensioneight operators and their running.

To conclude, let us remark that our results comprise one step further towards the one-loop renormalisation of the SMEFT to order $v^{4} / \Lambda^{4}$. This endeavor was initiated in Refs. [4 9] (see also Refs. 66 68] for the renormalisation of the Weinbeg operator) and continued in Ref. [69] (for baryon-number violating interactions), Ref. [30] (which includes the renormalisation of dimension-six operators by pairs of Weinberg interactions), Refs. 22, 70 (which involves the renormalisation of dimension-seven operators triggered by relevant couplings) and Ref. [71] (in which neutrino masses are renormalised to order $v^{3} / \Lambda^{3}$, including arbitrary combinations of dimension-five, -six and -seven operators). Some partial results of renormalisation within the dimension-eight sector of the SMEFT can be found in Refs. 19,72 . See Table 5 for a summary of the state of the art.

In forecoming works, we plan to extend the results of this paper with the inclusion, in the UV, of the operators of dimension eight that can be generated at tree level. We will also consider the renormalisation of non-bosonic operators. The latter can be induced, in particular, by field redefinitions aimed at removing the operators $\mathcal{O}_{B D \phi}, \mathcal{O}_{\phi D}^{\prime}, \mathcal{O}_{W D \phi}$ and $\mathcal{O}_{W \phi^{4} D^{2}}^{(6)}$; see Appendix A. Consequently, our current findings lay the basis for future work in this direction.

\section{Acknowledgments}

We would like to thank Jorge de Blas for useful discussions. MC and JS are supported by the Ministry of Science and Innovation under grant number FPA2016-78220-C3-1/3P (FEDER), SRA under grant PID2019-106087GB-C21/C22 (10.13039/501100011033), and by the Junta de Andalucía grants FQM 101, A-FQM-211-UGR18 and P18-FR-4314 (FEDER). MC is also supported by the Spanish MINECO under the Ramón y Cajal programme. GG and MR acknowledge support by LIP (FCT, COMPETE2020-Portugal2020, FEDER, POCI-01-0145-FEDER-007334) as well as by FCT under project CERN/FISPAR/0024/2019. GG is also supported by FCT under the grant SFRH/BD/144244/2019. MR is also supported by Fundação para a Ciência e Tecnologia (FCT) under the grant $\mathrm{PD} / \mathrm{BD} / 142773 / 2018$. 


\section{A Removing redundant operators}

The redundant operators generated in the process of renormalisation can be removed upon performing suitable perturbative field redefinitions, for example $\phi \rightarrow \phi+\frac{1}{\Lambda^{2}} \mathcal{O}$, where $\mathcal{O}$ is called the perturbation. We are interested in the effect of these field redefinitions to linear order in the perturbation (because $\mathcal{O}$ is loop suppressed and therefore quadratic powers of this term are formally two-loop corrections), which can be implemeted through the equations of motion of the SMEFT to order $v^{2} / \Lambda^{2}$ [53]. These read [73]:

$$
\begin{aligned}
D^{2} \phi^{i} & =-\mu^{2} \phi^{i}-2 \lambda\left(\phi^{\dagger} \phi\right) \phi^{i}+\frac{1}{\Lambda^{2}}\left\{3 c_{\phi}\left(\phi^{\dagger} \phi\right)^{2} \phi^{i}+2 c_{\phi \square} \phi^{i} \square\left(\phi^{\dagger} \phi\right)\right. \\
& \left.-c_{\phi D}\left[\left(D^{\mu} \phi\right)^{i}\left(\phi^{\dagger} \overleftrightarrow{D}_{\mu} \phi\right)+\phi^{i} \partial^{\mu}\left(\phi^{\dagger} D_{\mu} \phi\right)\right]\right\}+\cdots, \\
\partial^{\nu} B_{\mu \nu} & =\frac{g_{1}}{2} \phi^{\dagger} i \overleftrightarrow{D}_{\mu} \phi+\frac{c_{\phi D}}{\Lambda^{2}} \frac{g_{1}}{2}\left(\phi^{\dagger} \phi\right)\left(\phi^{\dagger} i \overleftrightarrow{D}_{\mu} \phi\right)+\cdots, \\
D^{\nu} W_{\mu \nu}^{I} & =\frac{g_{2}}{2} \phi^{\dagger} i \overleftrightarrow{D}_{\mu}^{I} \phi+\frac{c_{\phi D}}{\Lambda^{2}} g_{2}\left(\phi^{\dagger} \sigma^{I} \phi\right)\left(\phi^{\dagger} i \overleftrightarrow{D}_{\mu} \phi\right)+\cdots,
\end{aligned}
$$

where the ellipses represent fermionc operators, on which we are not interested. The following relations hold on-shell:

$$
\begin{aligned}
\mathcal{O}_{B D \phi} & =\frac{g_{1}}{2}\left[\mathcal{O}_{\phi \square}+4 \mathcal{O}_{\phi D}-\frac{\mu^{2}}{\Lambda^{2}} c_{\phi D} \mathcal{O}_{\phi}\right]+\frac{g_{1}}{2} \frac{c_{\phi D}}{\Lambda^{2}}\left[-2 \lambda \mathcal{O}_{\phi^{8}}+3 \mathcal{O}_{\phi^{6}}^{(1)}+2 \mathcal{O}_{\phi^{6}}^{(2)}\right]+\cdots, \\
\mathcal{O}_{\phi D}^{\prime} & =-\frac{1}{2}\left[-\mathcal{O}_{\phi \square}-2 \mu^{2}|\phi|^{4}-4 \lambda \mathcal{O}_{\phi}+\frac{\mu^{2}}{\Lambda^{2}}\left(c_{\phi D}-8 c_{\phi \square}\right) \mathcal{O}_{\phi}\right] \\
& -\frac{1}{2 \Lambda^{2}}\left[\left(6 c_{\phi}-16 \lambda c_{\phi \square}+2 \lambda c_{\phi D}\right) \mathcal{O}_{\phi^{8}}+\left(8 c_{\phi \square}+c_{\phi D}\right) \mathcal{O}_{\phi^{6}}^{(1)}+2 c_{\phi D} \mathcal{O}_{\phi^{6}}^{(2)}\right]+\cdots \\
\mathcal{O}_{W D \phi} & =-g_{2}\left[-\frac{3}{2} \mathcal{O}_{\phi \square}-2 \mu^{2}|\phi|^{4}-4 \lambda \mathcal{O}_{\phi}+\frac{\mu^{2}}{\Lambda^{2}}\left(2 c_{\phi D}-8 c_{\phi \square}\right) \mathcal{O}_{\phi}\right] \\
& -\frac{g_{2}}{\Lambda^{2}}\left[\left(6 c_{\phi}-16 \lambda c_{\phi \square}+4 \lambda c_{\phi D}\right) \mathcal{O}_{\phi^{8}}+\left(8 c_{\phi \square}-2 c_{\phi D}\right) \mathcal{O}_{\phi^{6}}^{(1)}\right]+\cdots
\end{aligned}
$$

where the ellipses encode again fermionic interactions. The operator $\mathcal{O}_{\phi D}^{\prime \prime}$ gives no contributions to the bosonic sector.

To arrive at these results, we used the following identities:

$$
\begin{aligned}
|\phi|^{2}\left(\phi^{\dagger} D_{\mu} \phi\right)\left(D^{\mu} \phi^{\dagger} \phi\right) & =\frac{1}{2}\left[\mathcal{O}_{\phi^{6}}^{(1)}+\mathcal{O}_{\phi^{6}}^{(2)}\right] \\
|\phi|^{2}\left(\phi^{\dagger} \mathrm{i} \overleftrightarrow{D} \phi\right)^{2} & =3 \mathcal{O}_{\phi^{6}}^{(1)}+2 \mathcal{O}_{\phi^{6}}^{(2)}+\frac{1}{2} \mathcal{O}_{\phi^{6}}^{(3)} \\
|\phi|^{4} \square|\phi|^{2} & =2 \mathcal{O}_{\phi^{6}}^{(1)}+\mathcal{O}_{\phi^{6}}^{(3)}
\end{aligned}
$$

In turn, the redundant dimension-eight operators become, on-shell:

$$
\begin{aligned}
& \mathcal{O}_{\phi^{6}}^{(3)}=-2 \mu^{2} \mathcal{O}_{\phi}-4 \lambda \mathcal{O}_{\phi^{8}} \\
& \mathcal{O}_{\phi^{4}}^{(4)}=\mu^{2}\left[-\mathcal{O}_{\phi D}-2 \mu^{2}|\phi|^{4}-4 \lambda \mathcal{O}_{\phi}\right]-4 \lambda \mathcal{O}_{\phi^{6}}^{(1)},
\end{aligned}
$$




$$
\begin{aligned}
\mathcal{O}_{\phi^{4}}^{(6)} & =-2 \mu^{2} \mathcal{O}_{\phi D}-2 \lambda\left[\mathcal{O}_{\phi^{6}}^{(1)}+\mathcal{O}_{\phi^{6}}^{(2)}\right] \\
\mathcal{O}_{\phi^{4}}^{(8)} & =2 \mu^{2}\left[\mu^{2}|\phi|^{4}+4 \lambda \mathcal{O}_{\phi}\right]+8 \lambda^{2} \mathcal{O}_{\phi^{8}} \\
\mathcal{O}_{\phi^{4}}^{(10)} & =\mu^{2}\left[\mu^{2}|\phi|^{4}+4 \lambda \mathcal{O}_{\phi}\right]+4 \lambda^{2} \mathcal{O}_{\phi^{8}} \\
\mathcal{O}_{\phi^{4}}^{(11)} & =\mu^{2}\left[\mu^{2}|\phi|^{4}+4 \lambda \mathcal{O}_{\phi}\right]+4 \lambda^{2} \mathcal{O}_{\phi^{8}} \\
\mathcal{O}_{\phi^{4}}^{(12)} & =\mu^{2}\left[2 \mathcal{O}_{\phi D}+\mathcal{O}_{\phi \square}-2 \lambda \mathcal{O}_{\phi}\right]+2\left[2 \lambda \mathcal{O}_{\phi^{6}}^{(1)}+\lambda \mathcal{O}_{\phi^{6}}^{(2)}-2 \lambda^{2} \mathcal{O}_{\phi^{8}}\right], \\
\mathcal{O}_{W \phi^{4} D^{2}}^{(6)} & =-\frac{g_{2}}{2}\left[-\mu^{2} \mathcal{O}_{\phi}+5 \mathcal{O}_{\phi^{6}}^{(1)}-2 \lambda \mathcal{O}_{\phi^{8}}+\cdots\right] \\
\mathcal{O}_{W \phi^{4} D^{2}}^{(7)} & =\frac{1}{4}\left\{\frac{g_{2}}{2}\left[2 \mu^{2} \mathcal{O}_{\phi}+\mathcal{O}_{W^{2} \phi^{4}}^{(1)}+\mathcal{O}_{W^{2} \phi^{4}}^{(3)}\right]+g_{1} \mathcal{O}_{W B \phi^{4}}^{(1)}+8 \mathcal{O}_{W \phi^{4} D^{2}}^{(1)}\right. \\
& \left.-6 g_{2} \mathcal{O}_{\phi^{6}}^{(1)}+g_{2} \mathcal{O}_{\phi^{6}}^{(2)}+2 g_{2} \lambda \mathcal{O}_{\phi^{8}}^{(1)}\right\} \\
\mathcal{O}_{B \phi^{4} D^{2}}^{(3)} & =-\frac{g_{1}}{2}\left[-\mu^{2} \mathcal{O}_{\phi}+3 \mathcal{O}_{\phi^{6}}^{(1)}+2 \mathcal{O}_{\phi^{6}}^{(2)}-2 \lambda \mathcal{O}_{\phi^{8}}\right]
\end{aligned}
$$

where again the ellipses represent terms on which we are not interested in this work. The operator $\mathcal{O}_{\phi^{6}}^{(4)}$ contributes only to fermionic interactions.

Altogether, these equations lead to:

$$
\begin{aligned}
\lambda & \rightarrow \lambda-\frac{\mu^{2}}{\Lambda^{2}} c_{\phi D}^{\prime}-\frac{\mu^{4}}{\Lambda^{4}}\left[-2\left(c_{\phi^{4}}^{(4)}-c_{\phi^{4}}^{(8)}\right)+c_{\phi^{4}}^{(10)}+c_{\phi^{4}}^{(11)}\right], \\
c_{\phi} & \rightarrow c_{\phi}+2 \lambda c_{\phi D}^{\prime}+\frac{\mu^{2}}{2 \Lambda^{4}}\left[3\left(c_{\phi D}+2 c_{\phi \square}\right) c_{\phi}-\left(c_{\phi D}-8 c_{\phi \square}\right)\left(c_{\phi D}^{\prime}+2 g_{2} c_{W D \phi}\right)\right. \\
& -2 g_{2} c_{\phi D} c_{W D \phi}-g_{1} c_{\phi D} c_{B D \phi}-4 c_{\phi^{6}}^{(3)}+4 \lambda\left\{-2 c_{\phi^{4}}^{(4)}+4 c_{\phi^{4}}^{(8)}+2 c_{\phi^{4}}^{(10)}+2 c_{\phi^{4}}^{(11)}-c_{\phi^{4}}^{(12)}\right\} \\
& \left.+g_{2} c_{W \phi^{4} D^{2}}^{(6)}+\frac{g_{2}}{2} c_{W \phi^{4} D^{2}}^{(7)}+g_{1} c_{B \phi^{4} D^{2}}^{(3)}\right], \\
c_{\phi D} & \rightarrow c_{\phi D}+2 \frac{\mu^{2}}{\Lambda^{4}}\left[\frac{1}{2}\left(c_{\phi D}+2 c_{\phi \square}\right) c_{\phi D}-c_{\phi^{4}}^{(6)}+c_{\phi^{4}}^{(12)}\right], \\
c_{\phi \square} & \rightarrow c_{\phi \square}+\frac{1}{2} c_{\phi D}^{\prime}+\frac{\mu^{2}}{\Lambda^{4}}\left[\left(c_{\phi D}+2 c_{\phi \square}\right) c_{\phi \square}-c_{\phi^{4}}^{(4)}+c_{\phi^{4}}^{(12)}\right],
\end{aligned}
$$

where we have already normalised canonically the Higgs kinetic term; as well as

$$
\begin{aligned}
c_{\phi^{8}} & \rightarrow c_{\phi^{8}}-g_{1} \lambda c_{B D \phi} c_{\phi D}-\left(c_{\phi D}^{\prime}+2 g_{2} c_{W D \phi}\right)\left(3 c_{\phi}-8 \lambda c_{\phi \square}+\lambda c_{\phi D}\right) \\
& -2 g_{2} \lambda c_{W D \phi} c_{\phi D}-4 \lambda c_{\phi^{6}}^{(3)}+4 \lambda^{2}\left[2 c_{\phi^{4}}^{(8)}+c_{\phi^{4}}^{(10)}+c_{\phi^{4}}^{(11)}-c_{\phi^{4}}^{(12)}\right] \\
& +g_{2} \lambda c_{W \phi^{4} D^{2}}^{(6)}+\frac{g_{2}}{2} \lambda c_{W \phi^{4} D^{2}}^{(7)}+g_{1} \lambda c_{B \phi^{4} D^{2}}^{(3)}, \\
c_{\phi^{6}}^{(1)} & \rightarrow c_{\phi^{6}}^{(1)}+\frac{3}{2} g_{1} c_{B D \phi} c_{\phi D}-\frac{\left(c_{\phi D}^{\prime}+2 g_{2} c_{W D \phi}\right)}{2}\left(8 c_{\phi \square}+c_{\phi D}\right)+3 g_{2} c_{W D \phi} c_{\phi D} \\
& -2 \lambda\left[2 c_{\phi^{4}}^{(4)}+c_{\phi^{4}}^{(6)}-2 c_{\phi^{4}}^{(12)}\right]-\frac{5}{2} g_{2} c_{W \phi^{4} D^{2}}^{(6)}-\frac{3}{2} g_{2} c_{W \phi^{4} D^{2}}^{(7)}-\frac{3}{2} g_{1} c_{B \phi^{4} D^{2}}^{(3)}, \\
c_{\phi^{6}}^{(2)} & \rightarrow c_{\phi^{6}}^{(2)}+g_{1} c_{B D \phi} c_{\phi D}-c_{\phi D^{\prime}}^{\prime} c_{\phi D}-2 \lambda\left[c_{\phi^{4}}^{(6)}-c_{\phi^{4}}^{(12)}\right]+\frac{g_{2}}{4} c_{W \phi^{4} D^{2}}^{(7)}-g_{1} c_{B \phi^{4} D^{2}}^{(3)},
\end{aligned}
$$




$$
\begin{aligned}
c_{W^{2} \phi^{4}}^{(1)} & \rightarrow c_{W^{2} \phi^{4}}^{(1)}+\frac{g_{2}}{8} c_{W \phi^{4} D^{2}}^{(7)}, \\
c_{W^{2} \phi^{4}}^{(3)} & \rightarrow c_{W^{2} \phi^{4}}^{(3)}+\frac{g_{2}}{8} c_{W \phi^{4} D^{2}}^{(7)}, \\
c_{W B \phi^{4}}^{(1)} & \rightarrow c_{W B \phi^{4}}^{(1)}+\frac{g_{1}}{4} c_{W \phi^{4} D^{2}}^{(7)} \\
c_{W \phi^{4} D^{2}}^{(1)} & \rightarrow c_{W \phi^{4} D^{2}}^{(1)}+2 c_{W \phi^{4} D^{2}}^{(7)} .
\end{aligned}
$$

311

\section{B Renormalisation group equations}

For a given coupling $c$, we define:

$$
\dot{c} \equiv 16 \pi^{2} \mu \frac{\mathrm{d} c}{d \mu} .
$$

310 Thus, we have:

$$
\dot{\lambda} \supset\left(5 c_{\phi D}^{2}-24 c_{\phi D} c_{\phi \square}+24 c_{\phi \square}^{2}\right) \frac{\mu^{4}}{\Lambda^{4}},
$$

$$
\begin{aligned}
& \dot{c}_{\phi} \supset\left\{\frac{-37}{24} g_{1}^{2} c_{\phi D}^{2}+\frac{5}{8} g_{2}^{2} c_{\phi D}^{2}-35 \lambda c_{\phi D}^{2}+\frac{26}{3} c_{\phi \square} g_{1}^{2} c_{\phi D}-\frac{113}{6} c_{\phi \square} g_{2}^{2} c_{\phi D}-51 c_{\phi} c_{\phi D}\right. \\
& +320 \lambda c_{\phi \square} c_{\phi D}-3 \operatorname{Tr}\left[c_{d \phi} y^{d \dagger}\right] c_{\phi D}-\operatorname{Tr}\left[c_{e \phi} y^{e \dagger}\right] c_{\phi D}-3 \operatorname{Tr}\left[c_{u \phi} y^{u \dagger}\right] c_{\phi D}-3 \operatorname{Tr}\left[\left(c_{d \phi}\right)^{\dagger} y^{d}\right] c_{\phi D} \\
& -\operatorname{Tr}\left[\left(c_{e \phi}\right)^{\dagger} y^{e}\right] c_{\phi D}-3 \operatorname{Tr}\left[\left(c_{u \phi}\right)^{\dagger} y^{u}\right] c_{\phi D}-6 \operatorname{Tr}\left[y^{u \dagger} y^{d}\left(c_{\phi u d}\right)^{\dagger}\right] c_{\phi D}+4 \operatorname{Tr}\left[c_{\phi l}^{(3)} y^{e} y^{e \dagger}\right] c_{\phi D} \\
& +12 \operatorname{Tr}\left[c_{\phi q}^{(3)} y^{d} y^{d \dagger}\right] c_{\phi D}+12 \operatorname{Tr}\left[c_{\phi q}^{(3)} y^{u} y^{u \dagger}\right] c_{\phi D}-6 \operatorname{Tr}\left[c_{\phi u d} y^{d \dagger} y^{u}\right] c_{\phi D}+\frac{2}{3} g_{1}^{2} \operatorname{Tr}\left[c_{\phi d}\right] c_{\phi D} \\
& +\frac{2}{3} g_{1}^{2} \operatorname{Tr}\left[c_{\phi e}\right] c_{\phi D}+\frac{2}{3} g_{1}^{2} \operatorname{Tr}\left[c_{\phi l}^{(1)}\right] c_{\phi D}-\frac{8}{3} g_{2}^{2} \operatorname{Tr}\left[c_{\phi l}^{(3)}\right] c_{\phi D}-\frac{2}{3} g_{1}^{2} \operatorname{Tr}\left[c_{\phi q}^{(1)}\right] c_{\phi D}-8 g_{2}^{2} \operatorname{Tr}\left[c_{\phi q}^{(3)}\right] c_{\phi D} \\
& -\frac{4}{3} g_{1}^{2} \operatorname{Tr}\left[c_{\phi u}\right] c_{\phi D}-640 \lambda c_{\phi \square}^{2}-\frac{20}{3} c_{\phi \square}^{2} g_{1}^{2}+20 c_{\phi \square}^{2} g_{2}^{2}+222 c_{\phi} c_{\phi \square}+24 c_{\phi \square} \operatorname{Tr}\left[c_{d \phi} y^{d \dagger}\right] \\
& +8 c_{\phi \square} \operatorname{Tr}\left[c_{e \phi} y^{e \dagger}\right]+24 c_{\phi \square} \operatorname{Tr}\left[c_{u \phi} y^{u \dagger}\right]+24 c_{\phi \square} \operatorname{Tr}\left[\left(c_{d \phi}\right)^{\dagger} y^{d}\right]-12 \operatorname{Tr}\left[\left(c_{d \phi}\right)^{\dagger} c_{d \phi}\right] \\
& +8 c_{\phi \square} \operatorname{Tr}\left[\left(c_{e \phi}\right)^{\dagger} y^{e}\right]-4 \operatorname{Tr}\left[\left(c_{e \phi}\right)^{\dagger} c_{e \phi}\right]+24 c_{\phi \square} \operatorname{Tr}\left[\left(c_{u \phi}\right)^{\dagger} y^{u}\right]-12 \operatorname{Tr}\left[\left(c_{u \phi}\right)^{\dagger} c_{u \phi}\right] \\
& -2 g_{2}^{2} \operatorname{Tr}\left[\left(c_{\phi d}\right)^{\dagger} c_{\phi d}\right]-\frac{2}{3} g_{2}^{2} \operatorname{Tr}\left[\left(c_{\phi e}\right)^{\dagger} c_{\phi e}\right]-\frac{4}{3} g_{2}^{2} \operatorname{Tr}\left[\left(c_{\phi l}^{(1)}\right)^{\dagger} c_{\phi l}^{(1)}\right]-\frac{8}{3} g_{1}^{2} \operatorname{Tr}\left[\left(c_{\phi l}^{(3)}\right)^{\dagger} c_{\phi l}^{(3)}\right] \\
& +\frac{4}{3} g_{2}^{2} \operatorname{Tr}\left[\left(c_{\phi l}^{(3)}\right)^{\dagger} c_{\phi l}^{(3)}\right]-4 g_{2}^{2} \operatorname{Tr}\left[\left(c_{\phi q}^{(1)}\right)^{\dagger} c_{\phi q}^{(1)}\right]-8 g_{1}^{2} \operatorname{Tr}\left[\left(c_{\phi q}^{(3)}\right)^{\dagger} c_{\phi q}^{(3)}\right]+4 g_{2}^{2} \operatorname{Tr}\left[\left(c_{\phi q}^{(3)}\right)^{\dagger} c_{\phi q}^{(3)}\right] \\
& -2 g_{2}^{2} \operatorname{Tr}\left[\left(c_{\phi u}\right)^{\dagger} c_{\phi u}\right]+g_{1}^{2} \operatorname{Tr}\left[\left(c_{\phi u d}\right)^{\dagger} c_{\phi u d}\right]-g_{2}^{2} \operatorname{Tr}\left[\left(c_{\phi u d}\right)^{\dagger} c_{\phi u d}\right]+48 c_{\phi \square} \operatorname{Tr}\left[y^{u \dagger} y^{d}\left(c_{\phi u d}\right)^{\dagger}\right] \\
& -6 \operatorname{Tr}\left[c_{d \phi} y^{d \dagger \dagger} c_{\phi q}^{(1)}\right]-6 \operatorname{Tr}\left[c_{d \phi} y^{d \dagger} c_{\phi q}^{(3)}\right]-2 \operatorname{Tr}\left[c_{e \phi} y^{e \dagger} c_{\phi l}^{(1)}\right]-2 \operatorname{Tr}\left[c_{e \phi} y^{e \dagger} c_{\phi l}^{(3)}\right]+6 \operatorname{Tr}\left[c_{u \phi} y^{u \dagger} c_{\phi q}^{(1)}\right] \\
& -6 \operatorname{Tr}\left[c_{u \phi} y^{u \dagger} c_{\phi q}^{(3)}\right]+6 \operatorname{Tr}\left[c_{\phi d} y^{d \dagger} c_{d \phi}\right]+2 \operatorname{Tr}\left[c_{\phi e} y^{e \dagger} c_{e \phi}\right]-2 \operatorname{Tr}\left[c_{\phi l}^{(1)} y^{e}\left(c_{e \phi}\right)^{\dagger}\right]-32 c_{\phi \square} \operatorname{Tr}\left[c_{\phi l}^{(3)} y^{e} y^{e \dagger}\right] \\
& -2 \operatorname{Tr}\left[c_{\phi l}^{(3)} y^{e}\left(c_{e \phi}\right)^{\dagger}\right]-6 \operatorname{Tr}\left[c_{\phi q}^{(1)} y^{d}\left(c_{d \phi}\right)^{\dagger}\right]+6 \operatorname{Tr}\left[c_{\phi q}^{(1)} y^{u}\left(c_{u \phi}\right)^{\dagger}\right]-96 c_{\phi \square} \operatorname{Tr}\left[c_{\phi q}^{(3)} y^{d} y^{d \dagger}\right] \\
& -6 \operatorname{Tr}\left[c_{\phi q}^{(3)} y^{d}\left(c_{d \phi}\right)^{\dagger}\right]-96 c_{\phi \square} \operatorname{Tr}\left[c_{\phi q}^{(3)} y^{u} y^{u \dagger}\right]-6 \operatorname{Tr}\left[c_{\phi q}^{(3)} y^{u}\left(c_{u \phi}\right)^{\dagger}\right]-6 \operatorname{Tr}\left[c_{\phi u} y^{u \dagger} c_{u \phi}\right] \\
& +48 c_{\phi \square} \operatorname{Tr}\left[c_{\phi u d} y^{d \dagger} y^{u}\right]+6 \operatorname{Tr}\left[\left(c_{d \phi}\right)^{\dagger} y^{d} c_{\phi d}\right]+2 \operatorname{Tr}\left[\left(c_{e \phi}\right)^{\dagger} y^{e} c_{\phi e}\right]-6 \operatorname{Tr}\left[\left(c_{u \phi}\right)^{\dagger} y^{u} c_{\phi u}\right] \\
& +6 \operatorname{Tr}\left[c_{\phi d} y^{d \dagger} y^{d} c_{\phi d}\right]+2 \operatorname{Tr}\left[c_{\phi e} y^{e \dagger} y^{e} c_{\phi e}\right]+2 \operatorname{Tr}\left[c_{\phi l}^{(1)} y^{e} y^{e \dagger} c_{\phi l}^{(1)}\right]+2 \operatorname{Tr}\left[c_{\phi l}^{(1)} y^{e} y^{e \dagger} c_{\phi l}^{(3)}\right] \\
& -4 \operatorname{Tr}\left[c_{\phi l}^{(1)} y^{e} c_{\phi e} y^{e \dagger}\right]+2 \operatorname{Tr}\left[c_{\phi l}^{(3)} y^{e} y^{e \dagger} c_{\phi l}^{(1)}\right]+2 \operatorname{Tr}\left[c_{\phi l}^{(3)} y^{e} y^{e \dagger} c_{\phi l}^{(3)}\right]-4 \operatorname{Tr}\left[c_{\phi l}^{(3)} y^{e} c_{\phi e} y^{e \dagger}\right] \\
& +6 \operatorname{Tr}\left[c_{\phi q}^{(1)} y^{d} y^{d \dagger} c_{\phi q}^{(1)}\right]+6 \operatorname{Tr}\left[c_{\phi q}^{(1)} y^{d} y^{d \dagger} c_{\phi q}^{(3)}\right]-12 \operatorname{Tr}\left[c_{\phi q}^{(1)} y^{d} c_{\phi d} y^{d \dagger}\right]+6 \operatorname{Tr}\left[c_{\phi q}^{(1)} y^{u} y^{u \dagger} c_{\phi q}^{(1)}\right]
\end{aligned}
$$




$$
\begin{aligned}
& -6 \operatorname{Tr}\left[c_{\phi q}^{(1)} y^{u} y^{u \dagger} c_{\phi q}^{(3)}\right]-12 \operatorname{Tr}\left[c_{\phi q}^{(1)} y^{u} c_{\phi u} y^{u \dagger}\right]+6 \operatorname{Tr}\left[c_{\phi q}^{(3)} y^{d} y^{d \dagger} c_{\phi q}^{(1)}\right]+6 \operatorname{Tr}\left[c_{\phi q}^{(3)} y^{d} y^{d \dagger} c_{\phi q}^{(3)}\right] \\
& -12 \operatorname{Tr}\left[c_{\phi q}^{(3)} y^{d} c_{\phi d} y^{d \dagger}\right]-6 \operatorname{Tr}\left[c_{\phi q}^{(3)} y^{u} y^{u \dagger} c_{\phi q}^{(1)}\right]+6 \operatorname{Tr}\left[c_{\phi q}^{(3)} y^{u} y^{u \dagger} c_{\phi q}^{(3)}\right]+12 \operatorname{Tr}\left[c_{\phi q}^{(3)} y^{u} c_{\phi u} y^{u \dagger}\right] \\
& \left.+6 \operatorname{Tr}\left[c_{\phi u} y^{u \dagger} y^{u} c_{\phi u}\right]+\frac{32}{3} c_{\phi \square} g_{2}^{2} \operatorname{Tr}\left[c_{\phi l}^{(3)}\right]+32 c_{\phi \square} g_{2}^{2} \operatorname{Tr}\left[c_{\phi q}^{(3)}\right]\right\} \frac{\mu^{2}}{\Lambda^{2}}, \\
& \dot{c}_{\phi D} \supset\left(-10 c_{\phi D}^{2}+4 c_{\phi D} c_{\phi \square}\right) \frac{\mu^{2}}{\Lambda^{2}}, \\
& \dot{c}_{\phi \square} \supset\left(-\frac{3}{2} c_{\phi D}^{2}-14 c_{\phi D} c_{\phi \square}+36 c_{\phi \square}^{2}\right) \frac{\mu^{2}}{\Lambda^{2}} . \\
& \dot{c}_{\phi^{8}}=-126 c_{\phi}^{2}-60 c_{\phi D}^{2} \lambda^{2}-864 c_{\phi \square}^{2} \lambda^{2}-4\left(c_{\phi D}^{2}-10 c_{\phi \square} c_{\phi D}+32 c_{\phi \square}^{2}\right) \lambda^{2} \\
& +48 c_{\phi}\left(15 c_{\phi D}+18 \lambda c_{\phi \square}-4 c_{\phi \square}\right) \lambda+\frac{1}{6} g_{2}^{2}\left(c_{\phi D}^{2}-12 c_{\phi \square} c_{\phi D}-24 \operatorname{Tr}\left[\left(c_{\phi d}\right)^{\dagger} c_{\phi d}\right]\right. \\
& -8 \operatorname{Tr}\left[\left(c_{\phi e}\right)^{\dagger} c_{\phi e}\right]-16 \operatorname{Tr}\left[\left(c_{\phi l}^{(1)}\right)^{\dagger} c_{\phi l}^{(1)}\right]-48 \operatorname{Tr}\left[\left(c_{\phi q}^{(1)}\right)^{\dagger} c_{\phi q}^{(1)}\right]-24 \operatorname{Tr}\left[\left(c_{\phi u}\right)^{\dagger} c_{\phi u}\right] \\
& \left.+12 \operatorname{Tr}\left[\left(c_{\phi u d}\right)^{\dagger} c_{\phi u d}\right]\right) \lambda+\frac{1}{12} g_{1}^{2}\left(3 c_{\phi D}^{2}-4 c_{\phi \square} c_{\phi D}-16 c_{\phi \square}^{2}-64 \operatorname{Tr}\left[\left(c_{\phi l}^{(3)}\right)^{\dagger} c_{\phi l}^{(3)}\right]\right. \\
& \left.-192 \operatorname{Tr}\left[\left(c_{\phi q}^{(3)}\right)^{\dagger} c_{\phi q}^{(3)}\right]+24 \operatorname{Tr}\left[\left(c_{\phi u d}\right)^{\dagger} c_{\phi u d}\right]\right) \lambda+\frac{1}{12} g_{2}^{2}\left(-5 c_{\phi D}^{2}+20 c_{\phi \square} c_{\phi D}-16 c_{\phi \square}^{2}\right. \\
& \left.+32 \operatorname{Tr}\left[\left(c_{\phi l}^{(3)}\right)^{\dagger} c_{\phi l}^{(3)}\right]+96 \operatorname{Tr}\left[\left(c_{\phi q}^{(3)}\right)^{\dagger} c_{\phi q}^{(3)}\right]-48 \operatorname{Tr}\left[\left(c_{\phi u d}\right)^{\dagger} c_{\phi u d}\right]\right) \lambda+\frac{1}{2}\left\{-3\left(g_{2}^{2}+4 \lambda\right) c_{\phi D}^{2}\right. \\
& -4 c_{\phi \square}\left(3 g_{1}^{2}+3 g_{2}^{2}-112 \lambda\right) c_{\phi D}-48 c_{\phi}\left(c_{\phi D}-9 c_{\phi \square}\right)-8 c_{\phi \square}^{2}\left(128 \lambda+3 g_{1}^{2}+3 g_{2}^{2}\right) \\
& +8\left(-6 \operatorname{Tr}\left[\left(c_{d \phi}\right)^{\dagger} c_{d \phi}\right]-2 \operatorname{Tr}\left[\left(c_{e \phi}\right)^{\dagger} c_{e \phi}\right]-6 \operatorname{Tr}\left[\left(c_{u \phi}\right)^{\dagger} c_{u \phi}\right]-3 \operatorname{Tr}\left[y^{d}\left(c_{d \phi}\right)^{\dagger} c_{\phi q}^{(1)}\right]\right. \\
& -3 \operatorname{Tr}\left[y^{d}\left(c_{d \phi}\right)^{\dagger} c_{\phi q}^{(3)}\right]+3 \operatorname{Tr}\left[y^{d \dagger} c_{d \phi} c_{\phi d}\right]-\operatorname{Tr}\left[y^{e}\left(c_{e \phi}\right)^{\dagger} c_{\phi l}^{(1)}\right]-\operatorname{Tr}\left[y^{e}\left(c_{e \phi}\right)^{\dagger} c_{\phi l}^{(3)}\right]+\operatorname{Tr}\left[y^{e \dagger} c_{e \phi} c_{\phi e}\right] \\
& -3 \operatorname{Tr}\left[y^{u} c_{\phi u}\left(c_{u \phi}\right)^{\dagger}\right]+3 \operatorname{Tr}\left[y^{u \dagger} c_{\phi q}^{(1)} c_{u \phi}\right]-3 \operatorname{Tr}\left[y^{u \dagger} c_{\phi q}^{(3)} c_{u \phi}\right]-3 \operatorname{Tr}\left[c_{u \phi} c_{\phi u} y^{u \dagger}\right]+3 \operatorname{Tr}\left[c_{\phi d}\left(c_{d \phi}\right)^{\dagger} y^{d}\right] \\
& +\operatorname{Tr}\left[c_{\phi e}\left(c_{e \phi}\right)^{\dagger} y^{e}\right]-\operatorname{Tr}\left[c_{\phi l}^{(1)} c_{e \phi} y^{e \dagger}\right]-\operatorname{Tr}\left[c_{\phi l}^{(3)} c_{e \phi} y^{e \dagger}\right]-3 \operatorname{Tr}\left[c_{\phi q}^{(1)} c_{d \phi} y^{d \dagger}\right]-3 \operatorname{Tr}\left[c_{\phi q}^{(3)} c_{d \phi} y^{d \dagger}\right] \\
& +3 \operatorname{Tr}\left[\left(c_{u \phi}\right)^{\dagger} c_{\phi q}^{(1)} y^{u}\right]-3 \operatorname{Tr}\left[\left(c_{u \phi}\right)^{\dagger} c_{\phi q}^{(3)} y^{u}\right]-6 \operatorname{Tr}\left[y^{d} c_{\phi d} y^{d \dagger} c_{\phi q}^{(1)}\right]-6 \operatorname{Tr}\left[y^{d} c_{\phi d} y^{d \dagger} c_{\phi q}^{(3)}\right] \\
& +3 \operatorname{Tr}\left[y^{d} c_{\phi d} c_{\phi d} y^{d \dagger}\right]-2 \operatorname{Tr}\left[y^{e} c_{\phi e} y^{e \dagger} c_{\phi l}^{(1)}\right]-2 \operatorname{Tr}\left[y^{e} c_{\phi e} y^{e \dagger} c_{\phi l}^{(3)}\right]+\operatorname{Tr}\left[y^{e} c_{\phi e} c_{\phi e} y^{e \dagger}\right] \\
& +\operatorname{Tr}\left[y^{e \dagger} c_{\phi l}^{(1)} c_{\phi l}^{(1)} y^{e}\right]+\operatorname{Tr}\left[y^{e \dagger} c_{\phi l}^{(1)} c_{\phi l}^{(3)} y^{e}\right]+\operatorname{Tr}\left[y^{e \dagger} c_{\phi l}^{(3)} c_{\phi l}^{(1)} y^{e}\right]+\operatorname{Tr}\left[y^{e \dagger} c_{\phi l}^{(3)} c_{\phi l}^{(3)} y^{e}\right] \\
& +3\left(\operatorname{Tr}\left[y^{d \dagger} c_{\phi q}^{(1)} c_{\phi q}^{(1)} y^{d}\right]+\operatorname{Tr}\left[y^{d \dagger} c_{\phi q}^{(1)} c_{\phi q}^{(3)} y^{d}\right]+\operatorname{Tr}\left[y^{d \dagger} c_{\phi q}^{(3)} c_{\phi q}^{(1)} y^{d}\right]+\operatorname{Tr}\left[y^{d \dagger} c_{\phi q}^{(3)} c_{\phi q}^{(3)} y^{d}\right]\right. \\
& +\operatorname{Tr}\left[y^{u} c_{\phi u} c_{\phi u} y^{u \dagger}\right]-2 \operatorname{Tr}\left[y^{u \dagger} c_{\phi q}^{(1)} y^{u} c_{\phi u}\right]+\operatorname{Tr}\left[y^{u \dagger} c_{\phi q}^{(1)} c_{\phi q}^{(1)} y^{u}\right]-\operatorname{Tr}\left[y^{u \dagger} c_{\phi q}^{(1)} c_{\phi q}^{(3)} y^{u}\right] \\
& \left.\left.\left.+2 \operatorname{Tr}\left[y^{u \dagger} c_{\phi q}^{(3)} y^{u} c_{\phi u}\right]-\operatorname{Tr}\left[y^{u \dagger} c_{\phi q}^{(3)} c_{\phi q}^{(1)} y^{u}\right]+\operatorname{Tr}\left[y^{u \dagger} c_{\phi q}^{(3)} c_{\phi q}^{(3)} y^{u}\right]\right)\right)\right\} \lambda \\
& -\frac{2}{3} c_{\phi D} g_{2}^{2}\left(c_{\phi \square}+4 \operatorname{Tr}\left[c_{\phi l}^{(3)}\right]+12 \operatorname{Tr}\left[c_{\phi q}^{(3)}\right]\right) \lambda-\frac{1}{3} c_{\phi D} g_{1}^{2}\left(c_{\phi D}+c_{\phi \square}-4 \operatorname{Tr}\left[c_{\phi d}\right]-4 \operatorname{Tr}\left[c_{\phi e}\right]\right. \\
& \left.-4 \operatorname{Tr}\left[c_{\phi l}^{(1)}\right]+4 \operatorname{Tr}\left[c_{\phi q}^{(1)}\right]+8 \operatorname{Tr}\left[c_{\phi u}\right]\right) \lambda-\frac{3}{8} c_{\phi D}^{2}\left(g_{1}^{2}+g_{2}^{2}\right)^{2}+6\left(\operatorname{Tr}\left[y^{d}\left(c_{d \phi}\right)^{\dagger} y^{d}\left(c_{d \phi}\right)^{\dagger}\right]\right. \\
& \left.+2 \operatorname{Tr}\left[y^{d}\left(c_{d \phi}\right)^{\dagger} c_{d \phi} y^{d \dagger}\right]+\operatorname{Tr}\left[y^{d \dagger} c_{d \phi} y^{d \dagger} c_{d \phi}\right]+2 \operatorname{Tr}\left[y^{d \dagger} c_{d \phi}\left(c_{d \phi}\right)^{\dagger} y^{d}\right]\right)+2 \operatorname{Tr}\left[y^{e}\left(c_{e \phi}\right)^{\dagger} y^{e}\left(c_{e \phi}\right)^{\dagger}\right] \\
& +4 \operatorname{Tr}\left[y^{e}\left(c_{e \phi}\right)^{\dagger} c_{e \phi} y^{e \dagger}\right]+2 \operatorname{Tr}\left[y^{e \dagger} c_{e \phi} y^{e \dagger} c_{e \phi}\right]+4 \operatorname{Tr}\left[y^{e \dagger} c_{e \phi}\left(c_{e \phi}\right)^{\dagger} y^{e}\right]+6\left(\operatorname{Tr}\left[y^{u}\left(c_{u \phi}\right)^{\dagger} y^{u}\left(c_{u \phi}\right)^{\dagger}\right]\right. \\
& \left.+2 \operatorname{Tr}\left[y^{u}\left(c_{u \phi}\right)^{\dagger} c_{u \phi} y^{u \dagger}\right]+\operatorname{Tr}\left[y^{u \dagger} c_{u \phi} y^{u \dagger} c_{u \phi}\right]+2 \operatorname{Tr}\left[y^{u \dagger} c_{u \phi}\left(c_{u \phi}\right)^{\dagger} y^{u}\right]\right)
\end{aligned}
$$




$$
\begin{aligned}
& -\frac{1}{3}\left(3 c_{\phi}+\lambda\left(c_{\phi D}-8 c_{\phi \square}\right)\right)\left(9 c_{\phi D} g_{1}^{2}-9 c_{\phi D} g_{2}^{2}+20 c_{\phi \square} g_{2}^{2}+12 \lambda\left(c_{\phi D}-2 c_{\phi \square}\right)\right. \\
& +18 \operatorname{Tr}\left[y^{d}\left(c_{d \phi}\right)^{\dagger}\right]+18 \operatorname{Tr}\left[y^{d \dagger} c_{d \phi}\right]+6 \operatorname{Tr}\left[y^{e}\left(c_{e \phi}\right)^{\dagger}\right]+6 \operatorname{Tr}\left[y^{e \dagger} c_{e \phi}\right]+18 \operatorname{Tr}\left[y^{u}\left(c_{u \phi}\right)^{\dagger}\right] \\
& +18 \operatorname{Tr}\left[y^{u \dagger} c_{u \phi}\right]+36 \operatorname{Tr}\left[y^{d}\left(c_{\phi u d}\right)^{\dagger} y^{u \dagger}\right]-72 \operatorname{Tr}\left[y^{d \dagger} c_{\phi q}^{(3)} y^{d}\right]-24 \operatorname{Tr}\left[y^{e \dagger} c_{\phi l}^{(3)} y^{e}\right]+36 \operatorname{Tr}\left[y^{u} c_{\phi u d} y^{d \dagger}\right] \\
& \left.-72 \operatorname{Tr}\left[y^{u \dagger} c_{\phi q}^{(3)} y^{u}\right]+8 g_{2}^{2}\left(\operatorname{Tr}\left[c_{\phi l}^{(3)}\right]+3 \operatorname{Tr}\left[c_{\phi q}^{(3)}\right]\right)\right), \\
& \dot{c}_{\phi^{6}}^{(1)}=-12 c_{\phi}\left(c_{\phi D}+8 c_{\phi \square}\right)+\frac{1}{24}\left(3 g_{1}^{2}+157 g_{2}^{2}-552 \lambda\right) c_{\phi D}^{2}-3 \operatorname{Tr}\left[y^{d}\left(c_{d \phi}\right)^{\dagger}\right] c_{\phi D} \\
& -3 \operatorname{Tr}\left[y^{d \dagger} c_{d \phi}\right] c_{\phi D}-\operatorname{Tr}\left[y^{e}\left(c_{e \phi}\right)^{\dagger}\right] c_{\phi D}-\operatorname{Tr}\left[y^{e \dagger} c_{e \phi}\right] c_{\phi D}-3 \operatorname{Tr}\left[y^{u}\left(c_{u \phi}\right)^{\dagger}\right] c_{\phi D} \\
& -3 \operatorname{Tr}\left[y^{u \dagger} c_{u \phi}\right] c_{\phi D}-6 \operatorname{Tr}\left[y^{d}\left(c_{\phi u d}\right)^{\dagger} y^{u \dagger}\right] c_{\phi D}+12 \operatorname{Tr}\left[y^{d \dagger} c_{\phi q}^{(3)} y^{d}\right] c_{\phi D}+4 \operatorname{Tr}\left[y^{e \dagger} c_{\phi l}^{(3)} y^{e}\right] c_{\phi D} \\
& -6 \operatorname{Tr}\left[y^{u} c_{\phi u d} y^{d \dagger}\right] c_{\phi D}-2 g_{1}^{2} \operatorname{Tr}\left[c_{\phi d}\right] c_{\phi D}-2 g_{1}^{2} \operatorname{Tr}\left[c_{\phi e}\right] c_{\phi D}-2 g_{1}^{2} \operatorname{Tr}\left[c_{\phi l}^{(1)}\right] c_{\phi D}+\frac{8}{3} g_{2}^{2} \operatorname{Tr}\left[c_{\phi l}^{(3)}\right] c_{\phi D} \\
& +2 g_{1}^{2} \operatorname{Tr}\left[c_{\phi q}^{(1)}\right] c_{\phi D}+8 g_{2}^{2} \operatorname{Tr}\left[c_{\phi q}^{(3)}\right] c_{\phi D}+4 g_{1}^{2} \operatorname{Tr}\left[c_{\phi u}\right] c_{\phi D}+\left(-2 g_{1}^{2}+\frac{29 g_{2}^{2}}{2}-64 \lambda\right) c_{\phi \square} c_{\phi D} \\
& +\frac{4}{3} c_{\phi \square}^{2}\left(15 g_{1}^{2}+5 g_{2}^{2}+264 \lambda\right)-24 c_{\phi \square} \operatorname{Tr}\left[y^{d}\left(c_{d \phi}\right)^{\dagger}\right]-24 c_{\phi \square} \operatorname{Tr}\left[y^{d \dagger} c_{d \phi}\right]-8 c_{\phi \square} \operatorname{Tr}\left[y^{e}\left(c_{e \phi}\right)^{\dagger}\right] \\
& -8 c_{\phi \square} \operatorname{Tr}\left[y^{e \dagger} c_{e \phi}\right]-24 c_{\phi \square} \operatorname{Tr}\left[y^{u}\left(c_{u \phi}\right)^{\dagger}\right]-24 c_{\phi \square} \operatorname{Tr}\left[y^{u \dagger} c_{u \phi}\right]+6 \operatorname{Tr}\left[\left(c_{d \phi}\right)^{\dagger} c_{d \phi}\right]+2 \operatorname{Tr}\left[\left(c_{e \phi}\right)^{\dagger} c_{e \phi}\right] \\
& +6 \operatorname{Tr}\left[\left(c_{u \phi}\right)^{\dagger} c_{u \phi}\right]+12 g_{2}^{2} \operatorname{Tr}\left[\left(c_{\phi d}\right)^{\dagger} c_{\phi d}\right]+4 g_{2}^{2} \operatorname{Tr}\left[\left(c_{\phi e}\right)^{\dagger} c_{\phi e}\right]+8 g_{2}^{2} \operatorname{Tr}\left[\left(c_{\phi l}^{(1)}\right)^{\dagger} c_{\phi l}^{(1)}\right]+8 g_{1}^{2} \operatorname{Tr}\left[\left(c_{\phi l}^{(3)}\right)^{\dagger} c_{\phi l}^{(3)}\right] \\
& -\frac{20}{3} g_{2}^{2} \operatorname{Tr}\left[\left(c_{\phi l}^{(3)}\right)^{\dagger} c_{\phi l}^{(3)}\right]+24 g_{2}^{2} \operatorname{Tr}\left[\left(c_{\phi q}^{(1)}\right)^{\dagger} c_{\phi q}^{(1)}\right]+24 g_{1}^{2} \operatorname{Tr}\left[\left(c_{\phi q}^{(3)}\right)^{\dagger} c_{\phi q}^{(3)}\right]-20 g_{2}^{2} \operatorname{Tr}\left[\left(c_{\phi q}^{(3)}\right)^{\dagger} c_{\phi q}^{(3)}\right] \\
& +12 g_{2}^{2} \operatorname{Tr}\left[\left(c_{\phi u}\right)^{\dagger} c_{\phi u}\right]-3 g_{1}^{2} \operatorname{Tr}\left[\left(c_{\phi u d}\right)^{\dagger} c_{\phi u d}\right]+4 g_{2}^{2} \operatorname{Tr}\left[\left(c_{\phi u d}\right)^{\dagger} c_{\phi u d}\right]+18 \operatorname{Tr}\left[y^{d}\left(c_{d \phi}\right)^{\dagger} c_{\phi q}^{(1)}\right] \\
& +30 \operatorname{Tr}\left[y^{d}\left(c_{d \phi}\right)^{\dagger} c_{\phi q}^{(3)}\right]-48 c_{\phi \square} \operatorname{Tr}\left[y^{d}\left(c_{\phi u d}\right)^{\dagger} y^{u \dagger}\right]-6 \operatorname{Tr}\left[y^{d}\left(c_{\phi u d}\right)^{\dagger}\left(c_{u \phi}\right)^{\dagger}\right]-18 \operatorname{Tr}\left[y^{d \dagger} c_{d \phi} c_{\phi d}\right] \\
& +96 c_{\phi \square} \operatorname{Tr}\left[y^{d \dagger} c_{\phi q}^{(3)} y^{d}\right]+6 \operatorname{Tr}\left[y^{e}\left(c_{e \phi}\right)^{\dagger} c_{\phi l}^{(1)}\right]+10 \operatorname{Tr}\left[y^{e}\left(c_{e \phi}\right)^{\dagger} c_{\phi l}^{(3)}\right]-6 \operatorname{Tr}\left[y^{e \dagger} c_{e \phi} c_{\phi e}\right] \\
& +32 c_{\phi \square} \operatorname{Tr}\left[y^{e \dagger} c_{\phi l}^{(3)} y^{e}\right]+18 \operatorname{Tr}\left[y^{u} c_{\phi u}\left(c_{u \phi}\right)^{\dagger}\right]-48 c_{\phi \square} \operatorname{Tr}\left[y^{u} c_{\phi u d} y^{d \dagger}\right]-6 \operatorname{Tr}\left[y^{u \dagger} c_{d \phi}\left(c_{\phi u d}\right)^{\dagger}\right] \\
& -18 \operatorname{Tr}\left[y^{u \dagger} c_{\phi q}^{(1)} c_{u \phi}\right]+12\left(c_{\phi D}+8 c_{\phi \square}\right) \operatorname{Tr}\left[y^{u \dagger} c_{\phi q}^{(3)} y^{u}\right]+30 \operatorname{Tr}\left[y^{u \dagger} c_{\phi q}^{(3)} c_{u \phi}\right]+18 \operatorname{Tr}\left[c_{u \phi} c_{\phi u} y^{u \dagger}\right] \\
& -6 \operatorname{Tr}\left[c_{u \phi} c_{\phi u d} y^{d \dagger}\right]-18 \operatorname{Tr}\left[c_{\phi d}\left(c_{d \phi}\right)^{\dagger} y^{d}\right]-6 \operatorname{Tr}\left[c_{\phi e}\left(c_{e \phi}\right)^{\dagger} y^{e}\right]+6 \operatorname{Tr}\left[c_{\phi l}^{(1)} c_{e \phi} y^{e \dagger}\right]+10 \operatorname{Tr}\left[c_{\phi l}^{(3)} c_{e \phi} y^{e \dagger}\right] \\
& +18 \operatorname{Tr}\left[c_{\phi q}^{(1)} c_{d \phi} y^{d \dagger}\right]+30 \operatorname{Tr}\left[c_{\phi q}^{(3)} c_{d \phi} y^{d \dagger}\right]-6 \operatorname{Tr}\left[c_{\phi u d}\left(c_{d \phi}\right)^{\dagger} y^{u}\right]-18 \operatorname{Tr}\left[\left(c_{u \phi}\right)^{\dagger} c_{\phi q}^{(1)} y^{u}\right] \\
& +30 \operatorname{Tr}\left[\left(c_{u \phi}\right)^{\dagger} c_{\phi q}^{(3)} y^{u}\right]+36 \operatorname{Tr}\left[y^{d} c_{\phi d} y^{d \dagger} c_{\phi q}^{(1)}\right]+36 \operatorname{Tr}\left[y^{d} c_{\phi d} y^{d \dagger} c_{\phi q}^{(3)}\right]-18 \operatorname{Tr}\left[y^{d} c_{\phi d} c_{\phi d} y^{d \dagger}\right] \\
& -3 \operatorname{Tr}\left[y^{d}\left(c_{\phi u d}\right)^{\dagger} c_{\phi u d} y^{d \dagger}\right]-18 \operatorname{Tr}\left[y^{d \dagger} c_{\phi q}^{(1)} c_{\phi q}^{(1)} y^{d}\right]-18 \operatorname{Tr}\left[y^{d \dagger} c_{\phi q}^{(1)} c_{\phi q}^{(3)} y^{d}\right]+12 \operatorname{Tr}\left[y^{d \dagger} c_{\phi q}^{(3)} y^{u} c_{\phi u d}\right] \\
& -18 \operatorname{Tr}\left[y^{d \dagger} c_{\phi q}^{(3)} c_{\phi q}^{(1)} y^{d}\right]-30 \operatorname{Tr}\left[y^{d \dagger} c_{\phi q}^{(3)} c_{\phi q}^{(3)} y^{d}\right]+12 \operatorname{Tr}\left[y^{e} c_{\phi e} y^{e \dagger} c_{\phi l}^{(1)}\right]+12 \operatorname{Tr}\left[y^{e} c_{\phi e} y^{e \dagger} c_{\phi l}^{(3)}\right] \\
& -6 \operatorname{Tr}\left[y^{e} c_{\phi e} c_{\phi e} y^{e \dagger}\right]-6 \operatorname{Tr}\left[y^{e \dagger} c_{\phi l}^{(1)} c_{\phi l}^{(1)} y^{e}\right]-6 \operatorname{Tr}\left[y^{e \dagger} c_{\phi l}^{(1)} c_{\phi l}^{(3)} y^{e}\right]-6 \operatorname{Tr}\left[y^{e \dagger} c_{\phi l}^{(3)} c_{\phi l}^{(1)} y^{e}\right] \\
& -10 \operatorname{Tr}\left[y^{e \dagger} c_{\phi l}^{(3)} c_{\phi l}^{(3)} y^{e}\right]-18 \operatorname{Tr}\left[y^{u} c_{\phi u} c_{\phi u} y^{u \dagger}\right]-3 \operatorname{Tr}\left[y^{u} c_{\phi u d}\left(c_{\phi u d}\right)^{\dagger} y^{u \dagger}\right]+36 \operatorname{Tr}\left[y^{u \dagger} c_{\phi q}^{(1)} y^{u} c_{\phi u}\right] \\
& -18 \operatorname{Tr}\left[y^{u \dagger} c_{\phi q}^{(1)} c_{\phi q}^{(1)} y^{u}\right]+18 \operatorname{Tr}\left[y^{u \dagger} c_{\phi q}^{(1)} c_{\phi q}^{(3)} y^{u}\right]+12 \operatorname{Tr}\left[y^{u \dagger} c_{\phi q}^{(3)} y^{d}\left(c_{\phi u d}\right)^{\dagger}\right]-36 \operatorname{Tr}\left[y^{u \dagger} c_{\phi q}^{(3)} y^{u} c_{\phi u}\right] \\
& +18 \operatorname{Tr}\left[y^{u \dagger} c_{\phi q}^{(3)} c_{\phi q}^{(1)} y^{u}\right]-30 \operatorname{Tr}\left[y^{u \dagger} c_{\phi q}^{(3)} c_{\phi q}^{(3)} y^{u}\right]-\frac{32}{3} c_{\phi \square} g_{2}^{2} \operatorname{Tr}\left[c_{\phi l}^{(3)}\right]-32 c_{\phi \square} g_{2}^{2} \operatorname{Tr}\left[c_{\phi q}^{(3)}\right], \\
& \dot{c}_{\phi^{6}}^{(2)}=-\frac{2}{3}\left(3 \operatorname{Tr}\left[\left(c_{\phi d}\right)^{\dagger} c_{\phi d}\right]+\operatorname{Tr}\left[\left(c_{\phi e}\right)^{\dagger} c_{\phi e}\right]+2 \operatorname{Tr}\left[\left(c_{\phi l}^{(1)}\right)^{\dagger} c_{\phi l}^{(1)}\right]+6 \operatorname{Tr}\left[\left(c_{\phi q}^{(1)}\right)^{\dagger} c_{\phi q}^{(1)}\right]\right) g_{2}^{2} \\
& -18 c_{\phi} c_{\phi D}+\frac{1}{12}\left(\left(-35 g_{1}^{2}+46 g_{2}^{2}-312 \lambda\right) c_{\phi D}^{2}+4 c_{\phi \square}\left(20 g_{1}^{2}+15 g_{2}^{2}+192 \lambda\right) c_{\phi D}+160 c_{\phi \square}^{2} g_{1}^{2}\right) \\
& +12 \operatorname{Tr}\left[y^{d}\left(c_{d \phi}\right)^{\dagger} c_{\phi q}^{(1)}\right]+6 \operatorname{Tr}\left[y^{d}\left(c_{\phi u d}\right)^{\dagger}\left(c_{u \phi}\right)^{\dagger}\right]-12 \operatorname{Tr}\left[y^{d \dagger} c_{d \phi} c_{\phi d}\right]+4 \operatorname{Tr}\left[y^{e}\left(c_{e \phi}\right)^{\dagger} c_{\phi l}^{(1)}\right]
\end{aligned}
$$




$$
\begin{aligned}
& -4 \operatorname{Tr}\left[y^{e \dagger} c_{e \phi} c_{\phi e}\right]+12 \operatorname{Tr}\left[y^{u} c_{\phi u}\left(c_{u \phi}\right)^{\dagger}\right]+6 \operatorname{Tr}\left[y^{u \dagger} c_{d \phi}\left(c_{\phi u d}\right)^{\dagger}\right]-12 \operatorname{Tr}\left[y^{u \dagger} c_{\phi q}^{(1)} c_{u \phi}\right] \\
& +12 \operatorname{Tr}\left[c_{u \phi} c_{\phi u} y^{u \dagger}\right]+6 \operatorname{Tr}\left[c_{u \phi} c_{\phi u d} y^{d \dagger}\right]-12 \operatorname{Tr}\left[c_{\phi d}\left(c_{d \phi}\right)^{\dagger} y^{d}\right]-4 \operatorname{Tr}\left[c_{\phi e}\left(c_{e \phi}\right)^{\dagger} y^{e}\right]+4 \operatorname{Tr}\left[c_{\phi l}^{(1)} c_{e \phi} y^{e \dagger}\right] \\
& +12 \operatorname{Tr}\left[c_{\phi q}^{(1)} c_{d \phi} y^{d \dagger}\right]+6 \operatorname{Tr}\left[c_{\phi u d}\left(c_{d \phi}\right)^{\dagger} y^{u}\right]-12 \operatorname{Tr}\left[\left(c_{u \phi}\right)^{\dagger} c_{\phi q}^{(1)} y^{u}\right]+24 \operatorname{Tr}\left[y^{d} c_{\phi d} y^{d \dagger} c_{\phi q}^{(1)}\right] \\
& +24 \operatorname{Tr}\left[y^{d} c_{\phi d} y^{d \dagger} c_{\phi q}^{(3)}\right]-12 \operatorname{Tr}\left[y^{d} c_{\phi d} c_{\phi d} y^{d \dagger}\right]+3 \operatorname{Tr}\left[y^{d}\left(c_{\phi u d}\right)^{\dagger} c_{\phi u d} y^{d \dagger}\right]-12 \operatorname{Tr}\left[y^{d \dagger} c_{\phi q}^{(1)} c_{\phi q}^{(1)} y^{d}\right] \\
& -12 \operatorname{Tr}\left[y^{d \dagger} c_{\phi q}^{(1)} c_{\phi q}^{(3)} y^{d}\right]-12 \operatorname{Tr}\left[y^{d \dagger} c_{\phi q}^{(3)} y^{u} c_{\phi u d}\right]-12 \operatorname{Tr}\left[y^{d \dagger} c_{\phi q}^{(3)} c_{\phi q}^{(1)} y^{d}\right]+8 \operatorname{Tr}\left[y^{e} c_{\phi e} y^{e \dagger} c_{\phi l}^{(1)}\right] \\
& +8 \operatorname{Tr}\left[y^{e} c_{\phi e} y^{e \dagger} c_{\phi l}^{(3)}\right]-4 \operatorname{Tr}\left[y^{e} c_{\phi e} c_{\phi e} y^{e \dagger}\right]-4 \operatorname{Tr}\left[y^{e \dagger} c_{\phi l}^{(1)} c_{\phi l}^{(1)} y^{e}\right]-4 \operatorname{Tr}\left[y^{e \dagger} c_{\phi l}^{(1)} c_{\phi l}^{(3)} y^{e}\right] \\
& -4 \operatorname{Tr}\left[y^{e \dagger} c_{\phi l}^{(3)} c_{\phi l}^{(1)} y^{e}\right]-12 \operatorname{Tr}\left[y^{u} c_{\phi u} c_{\phi u} y^{u \dagger}\right]+3 \operatorname{Tr}\left[y^{u} c_{\phi u d}\left(c_{\phi u d}\right)^{\dagger} y^{u \dagger}\right]+24 \operatorname{Tr}\left[y^{u \dagger} c_{\phi q}^{(1)} y^{u} c_{\phi u}\right] \\
& -12 \operatorname{Tr}\left[y^{u \dagger} c_{\phi q}^{(1)} c_{\phi q}^{(1)} y^{u}\right]+12 \operatorname{Tr}\left[y^{u \dagger} c_{\phi q}^{(1)} c_{\phi q}^{(3)} y^{u}\right]-12 \operatorname{Tr}\left[y^{u \dagger} c_{\phi q}^{(3)} y^{d}\left(c_{\phi u d}\right)^{\dagger}\right]-24 \operatorname{Tr}\left[y^{u \dagger} c_{\phi q}^{(3)} y^{u} c_{\phi u}\right] \\
& +12 \operatorname{Tr}\left[y^{u \dagger} c_{\phi q}^{(3)} c_{\phi q}^{(1)} y^{u}\right]+\frac{1}{3}\left(16 \operatorname{Tr}\left[\left(c_{\phi l}^{(3)}\right)^{\dagger} c_{\phi l}^{(3)}\right] g_{1}^{2}+48 \operatorname{Tr}\left[\left(c_{\phi q}^{(3)}\right)^{\dagger} c_{\phi q}^{(3)}\right] g_{1}^{2}-6 \operatorname{Tr}\left[\left(c_{\phi u d}\right)^{\dagger} c_{\phi u d}\right] g_{1}^{2}\right. \\
& -4 c_{\phi D} \operatorname{Tr}\left[c_{\phi d}\right] g_{1}^{2}-4 c_{\phi D} \operatorname{Tr}\left[c_{\phi e}\right] g_{1}^{2}-4 c_{\phi D} \operatorname{Tr}\left[c_{\phi l}^{(1)}\right] g_{1}^{2}+4 c_{\phi D} \operatorname{Tr}\left[c_{\phi q}^{(1)}\right] g_{1}^{2}+8 c_{\phi D} \operatorname{Tr}\left[c_{\phi u}\right] g_{1}^{2} \\
& -18 c_{\phi D} \operatorname{Tr}\left[y^{d}\left(c_{d \phi}\right)^{\dagger}\right]-18 c_{\phi D} \operatorname{Tr}\left[y^{d \dagger} c_{d \phi}\right]-6 c_{\phi D} \operatorname{Tr}\left[y^{e}\left(c_{e \phi}\right)^{\dagger}\right]-6 c_{\phi D} \operatorname{Tr}\left[y^{e \dagger} c_{e \phi}\right] \\
& -18 c_{\phi D} \operatorname{Tr}\left[y^{u}\left(c_{u \phi}\right)^{\dagger}\right]-18 c_{\phi D} \operatorname{Tr}\left[y^{u \dagger} c_{u \phi}\right]-6 g_{2}^{2} \operatorname{Tr}\left[\left(c_{\phi u}\right)^{\dagger} c_{\phi u}\right]+3 g_{2}^{2} \operatorname{Tr}\left[\left(c_{\phi u d}\right)^{\dagger} c_{\phi u d}\right] \\
& -36 c_{\phi D} \operatorname{Tr}\left[y^{d}\left(c_{\phi u d}\right)^{\dagger} y^{u \dagger}\right]+72 c_{\phi D} \operatorname{Tr}\left[y^{d \dagger} c_{\phi q}^{(3)} y^{d}\right]+24 c_{\phi D} \operatorname{Tr}\left[y^{e \dagger} c_{\phi l}^{(3)} y^{e}\right]-36 c_{\phi D} \operatorname{Tr}\left[y^{u} c_{\phi u d} y^{d \dagger}\right] \\
& \left.+72 c_{\phi D} \operatorname{Tr}\left[y^{u \dagger} c_{\phi q}^{(3)} y^{u}\right]\right) \text {, } \\
& \dot{c}_{\phi^{4}}^{(1)}=\frac{1}{3}\left(24 \operatorname{Tr}\left[\left(c_{\phi d}\right)^{\dagger} c_{\phi d}\right]+8 \operatorname{Tr}\left[\left(c_{\phi e}\right)^{\dagger} c_{\phi e}\right]+16 \operatorname{Tr}\left[\left(c_{\phi l}^{(1)}\right)^{\dagger} c_{\phi l}^{(1)}\right]-16 \operatorname{Tr}\left[\left(c_{\phi l}^{(3)}\right)^{\dagger} c_{\phi l}^{(3)}\right]\right. \\
& +48 \operatorname{Tr}\left[\left(c_{\phi q}^{(1)}\right)^{\dagger} c_{\phi q}^{(1)}\right]-48 \operatorname{Tr}\left[\left(c_{\phi q}^{(3)}\right)^{\dagger} c_{\phi q}^{(3)}\right]+24 \operatorname{Tr}\left[\left(c_{\phi u}\right)^{\dagger} c_{\phi u}\right]-24 \operatorname{Tr}\left[\left(c_{\phi u d}\right)^{\dagger} c_{\phi u d}\right]+32 c_{\phi D} c_{\phi \square} \\
& \left.-11 c_{\phi D}^{2}-16 c_{\phi \square}^{2}\right) \text {, } \\
& \dot{c}_{\phi^{4}}^{(2)}=\frac{1}{3}\left(-8\left(3 \operatorname{Tr}\left[\left(c_{\phi d}\right)^{\dagger} c_{\phi d}\right]+\operatorname{Tr}\left[\left(c_{\phi e}\right)^{\dagger} c_{\phi e}\right]+2 \operatorname{Tr}\left[\left(c_{\phi l}^{(1)}\right)^{\dagger} c_{\phi l}^{(1)}\right]+2 \operatorname{Tr}\left[\left(c_{\phi l}^{(3)}\right)^{\dagger} c_{\phi l}^{(3)}\right]\right.\right. \\
& \left.\left.+6 \operatorname{Tr}\left[\left(c_{\phi q}^{(1)}\right)^{\dagger} c_{\phi q}^{(1)}\right]+6 \operatorname{Tr}\left[\left(c_{\phi q}^{(3)}\right)^{\dagger} c_{\phi q}^{(3)}\right]+3 \operatorname{Tr}\left[\left(c_{\phi u}\right)^{\dagger} c_{\phi u}\right]\right)-16 c_{\phi D} c_{\phi \square}-5 c_{\phi D}^{2}-16 c_{\phi \square}^{2}\right), \\
& \dot{c}_{\phi^{4}}^{(3)}=\frac{1}{3}\left(32 \operatorname{Tr}\left[\left(c_{\phi l}^{(3)}\right)^{\dagger} c_{\phi l}^{(3)}\right]+96 \operatorname{Tr}\left[\left(c_{\phi q}^{(3)}\right)^{\dagger} c_{\phi q}^{(3)}\right]+24 \operatorname{Tr}\left[\left(c_{\phi u d}\right)^{\dagger} c_{\phi u d}\right]-16 c_{\phi D} c_{\phi \square}\right. \\
& \left.+7 c_{\phi D}^{2}-40 c_{\phi \square}^{2}\right), \\
& \dot{c}_{W^{2} \phi^{4}}^{(1)}=-\frac{1}{24} g_{2}^{2}\left(3 c_{\phi D}^{2}+24 \operatorname{Tr}\left[\left(c_{\phi d}\right)^{\dagger} c_{\phi d}\right]-48 \operatorname{Tr}\left[\left(c_{\phi l}^{(3)}\right)^{\dagger} c_{\phi l}^{(3)}\right]+48 \operatorname{Tr}\left[\left(c_{\phi q}^{(1)}\right)^{\dagger} c_{\phi q}^{(1)}\right]\right. \\
& -144 \operatorname{Tr}\left[\left(c_{\phi q}^{(3)}\right)^{\dagger} c_{\phi q}^{(3)}\right]+24 \operatorname{Tr}\left[\left(c_{\phi u}\right)^{\dagger} c_{\phi u}\right]+24 \operatorname{Tr}\left[\left(c_{\phi u d}\right)^{\dagger} c_{\phi u d}\right]+8 \operatorname{Tr}\left[\left(c_{\phi e}\right)^{\dagger} c_{\phi e}\right] \\
& \left.+16 \operatorname{Tr}\left[\left(c_{\phi l}^{(1)}\right)^{\dagger} c_{\phi l}^{(1)}\right]\right) \\
& \dot{c}_{W \phi^{4} D^{2}}^{(1)}=-\frac{1}{3} g_{2}\left(3 c_{\phi D}^{2}+24 \operatorname{Tr}\left[\left(c_{\phi d}\right)^{\dagger} c_{\phi d}\right]-48 \operatorname{Tr}\left[\left(c_{\phi l}^{(3)}\right)^{\dagger} c_{\phi l}^{(3)}\right]+48 \operatorname{Tr}\left[\left(c_{\phi q}^{(1)}\right)^{\dagger} c_{\phi q}^{(1)}\right]\right. \\
& -144 \operatorname{Tr}\left[\left(c_{\phi q}^{(3)}\right)^{\dagger} c_{\phi q}^{(3)}\right]+24 \operatorname{Tr}\left[\left(c_{\phi u}\right)^{\dagger} c_{\phi u}\right]+24 \operatorname{Tr}\left[\left(c_{\phi u d}\right)^{\dagger} c_{\phi u d}\right]+8 \operatorname{Tr}\left[\left(c_{\phi e}\right)^{\dagger} c_{\phi e}\right] \\
& \left.+16 \operatorname{Tr}\left[\left(c_{\phi l}^{(1)}\right)^{\dagger} c_{\phi l}^{(1)}\right]\right)
\end{aligned}
$$




$$
\begin{aligned}
\dot{c}_{B^{2} \phi^{4}}^{(1)} & =\frac{1}{24} g_{1}^{2}\left(3 c_{\phi D}^{2}+24 \operatorname{Tr}\left[\left(c_{\phi d}\right)^{\dagger} c_{\phi d}\right]-48 \operatorname{Tr}\left[\left(c_{\phi l}^{(3)}\right)^{\dagger} c_{\phi l}^{(3)}\right]+48 \operatorname{Tr}\left[\left(c_{\phi q}^{(1)}\right)^{\dagger} c_{\phi q}^{(1)}\right]\right. \\
& -144 \operatorname{Tr}\left[\left(c_{\phi q}^{(3)}\right)^{\dagger} c_{\phi q}^{(3)}\right]+24 \operatorname{Tr}\left[\left(c_{\phi u}\right)^{\dagger} c_{\phi u}\right]+24 \operatorname{Tr}\left[\left(c_{\phi u d}\right)^{\dagger} c_{\phi u d}\right]+8 \operatorname{Tr}\left[\left(c_{\phi e}\right)^{\dagger} c_{\phi e}\right] \\
& \left.+16 \operatorname{Tr}\left[\left(c_{\phi l}^{(1)}\right)^{\dagger} c_{\phi l}^{(1)}\right]\right), \\
\dot{c}_{B \phi^{4} D^{2}}^{(1)} & =\frac{1}{3} g_{1}\left(3 c_{\phi D}^{2}+24 \operatorname{Tr}\left[\left(c_{\phi d}\right)^{\dagger} c_{\phi d}\right]-48 \operatorname{Tr}\left[\left(c_{\phi l}^{(3)}\right)^{\dagger} c_{\phi l}^{(3)}\right]+48 \operatorname{Tr}\left[\left(c_{\phi q}^{(1)}\right)^{\dagger} c_{\phi q}^{(1)}\right]\right. \\
& -144 \operatorname{Tr}\left[\left(c_{\phi q}^{(3)}\right)^{\dagger} c_{\phi q}^{(3)}\right]+24 \operatorname{Tr}\left[\left(c_{\phi u}\right)^{\dagger} c_{\phi u}\right]+24 \operatorname{Tr}\left[\left(c_{\phi u d}\right)^{\dagger} c_{\phi u d}\right]+8 \operatorname{Tr}\left[\left(c_{\phi e}\right)^{\dagger} c_{\phi e}\right] \\
& \left.+16 \operatorname{Tr}\left[\left(c_{\phi l}^{(1)}\right)^{\dagger} c_{\phi l}^{(1)}\right]\right) .
\end{aligned}
$$

\section{Ultraviolet completion of the Standard Model}

The purpose of this appendix is proving that there exists at least one UV completion of the SM that induces arbitrary values of $c_{\phi}, c_{\phi D}$ and $c_{\phi} \square$. To this aim, let us extend the SM (for $\mu^{2}=0$ ) with three colorless scalars: $\mathcal{S} \sim(1,1)_{0}, \Xi_{0} \sim(1,3)_{0}$ and $\Xi_{1} \sim(1,3)_{1}$. The numbers in parentheses and the subscript indicate the representations of $S U(3)_{c}, S U(2)_{L}$ and $U(1)_{Y}$, respectively.

Let us assume that they all have mass $M$ much larger than the EW scale, and that the new physics interaction Lagrangian is:

$$
\mathcal{L}_{\mathrm{NP}}=\kappa_{\mathcal{S}} \mathcal{S} \phi^{\dagger} \phi+\lambda_{\mathcal{S}} \mathcal{S}^{2} \phi^{\dagger} \phi+\kappa_{\Xi_{0}} \phi^{\dagger} \Xi_{0}^{a} \sigma_{a} \phi+\left(\kappa_{\Xi_{1}} \Xi_{1}^{a \dagger} \tilde{\phi}^{\dagger} \sigma_{a} \phi+\text { h.c. }\right) .
$$

(Other triple and quartic terms are allowed, but we just ignored them for simplicity.) Then, by integrating out the heavy modes at tree level at the scale $M$, we obtain [46]:

$$
\begin{aligned}
\frac{c_{\phi}}{\Lambda^{2}} & =-\lambda_{\mathcal{S}} \frac{\kappa_{\mathcal{S}}^{2}}{M^{4}}, \\
\frac{c_{\phi D}}{\Lambda^{2}} & =\frac{2}{M^{4}}\left(2 \kappa_{\Xi_{1}}^{2}-\kappa_{\Xi_{0}}^{2}\right), \\
\frac{c_{\phi \square}}{\Lambda^{2}} & =\frac{1}{2 M^{4}}\left(4 \kappa_{\Xi_{1}}^{2}+\kappa_{\Xi_{0}}^{2}-\kappa_{\mathcal{S}}^{2}\right) .
\end{aligned}
$$

Obviously, $c_{\phi}$ can have arbitrary sign by just tuning $\lambda_{\mathcal{S}}$. Likewise, $c_{\phi D}$ can be made arbitrarily negative provided $\kappa_{\Xi_{1}} / \kappa_{\Xi_{0}} \ll 1$, and positive otherwise. Notwithstanding this later choice, $c_{\phi \square}$ will be positive for small enough $\kappa_{\mathcal{S}}$ and negative for large values of this parameter. In summary, the signs of the three tree-level generated dimension-six operators are arbitrary and uncorrelated.

In the process of integrating out the fields of mass $M$, dimension-eight operators arise too. With the help of MatchingTools [74], we find that (see also Ref. [33]):

$$
\frac{c_{\phi^{4}}^{(1)}}{\Lambda^{4}}=4 \frac{\kappa_{\Xi_{0}}^{2}}{M^{6}}, \quad \frac{c_{\phi^{4}}^{(2)}}{\Lambda^{4}}=8 \frac{\kappa_{\Xi_{1}}^{2}}{M^{6}}, \quad \frac{c_{\phi^{4}}^{(3)}}{\Lambda^{4}}=\frac{2}{M^{6}}\left(\kappa_{\mathcal{S}}^{2}-\kappa_{\Xi_{0}}^{2}\right) .
$$

Contrary to the dimension-six Wilson coefficients above, these couplings fullfill the positivity bounds $c_{\phi^{4}}^{(2)} \geq 0, c_{\phi^{4}}^{(1)}+c_{\phi^{4}}^{(2)} \geq 0$ and $c_{\phi^{4}}^{(1)}+c_{\phi^{4}}^{(2)}+c_{\phi^{4}}^{(3)} \geq 0$ obtained in Ref. 33 for arbitrary values of the $\kappa$ s. 


\section{References}

[1] I. Brivio and M. Trott, The Standard Model as an Effective Field Theory, Phys. Rept. 793, 1 (2019), doi:10.1016/j.physrep.2018.11.002, 1706.08945.

[2] L. Yuan, Exotics and BSM in ATLAS and CMS (Non dark matter searches), PoS CORFU2019, 051 (2020), doi:10.22323/1.376.0051.

[3] J. Ellis, M. Madigan, K. Mimasu, V. Sanz and T. You, Top, Higgs, Diboson and Electroweak Fit to the Standard Model Effective Field Theory, JHEP 04, 279 (2021), doi:10.1007/JHEP04(2021)279, 2012.02779.

[4] C. Grojean, E. E. Jenkins, A. V. Manohar and M. Trott, Renormalization Group

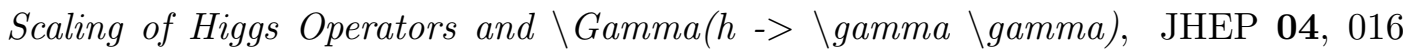
(2013), doi:10.1007/JHEP04(2013)016, 1301.2588.

[5] J. Elias-Miró, J. R. Espinosa, E. Masso and A. Pomarol, Renormalization of dimension-six operators relevant for the Higgs decays $h \rightarrow \gamma \gamma, \gamma Z$, JHEP 08, 033 (2013), doi:10.1007/JHEP08(2013)033, 1302.5661.

[6] J. Elias-Miro, J. R. Espinosa, E. Masso and A. Pomarol, Higgs windows to new physics through $d=6$ operators: constraints and one-loop anomalous dimensions, JHEP 11, 066 (2013), doi:10.1007/JHEP11(2013)066, 1308.1879.

[7] E. E. Jenkins, A. V. Manohar and M. Trott, Renormalization Group Evolution of the Standard Model Dimension Six Operators I: Formalism and lambda Dependence, JHEP 10, 087 (2013), doi:10.1007/JHEP10(2013)087, 1308.2627.

[8] E. E. Jenkins, A. V. Manohar and M. Trott, Renormalization Group Evolution of the Standard Model Dimension Six Operators II: Yukawa Dependence, JHEP 01, 035 (2014), doi:10.1007/JHEP01(2014)035, 1310.4838.

[9] R. Alonso, E. E. Jenkins, A. V. Manohar and M. Trott, Renormalization Group Evolution of the Standard Model Dimension Six Operators III: Gauge Coupling Dependence and Phenomenology, JHEP 04, 159 (2014), doi:10.1007/JHEP04(2014)159, 1312.2014.

[10] G. Buchalla, A. Celis, C. Krause and J.-N. Toelstede, Master Formula for One-Loop Renormalization of Bosonic SMEFT Operators (2019), 1904.07840.

[11] S. Descotes-Genon, A. Falkowski, M. Fedele, M. González-Alonso and J. Virto, The CKM parameters in the SMEFT, JHEP 05, 172 (2019), doi:10.1007/JHEP05(2019)172, 1812.08163.

[12] S. Bißmann, J. Erdmann, C. Grunwald, G. Hiller and K. Kröninger, Constraining top-quark couplings combining top-quark and $\boldsymbol{B}$ decay observables, Eur. Phys. J. C 80(2), 136 (2020), doi:10.1140/epjc/s10052-020-7680-9, 1909.13632.

[13] J. Terol-Calvo, M. Tórtola and A. Vicente, High-energy constraints from lowenergy neutrino nonstandard interactions, Phys. Rev. D 101(9), 095010 (2020), doi:10.1103/PhysRevD.101.095010, 1912.09131.

[14] C. Degrande, G. Durieux, F. Maltoni, K. Mimasu, E. Vryonidou and C. Zhang, Automated one-loop computations in the standard model effective field theory, Phys. Rev. D 103(9), 096024 (2021), doi:10.1103/PhysRevD.103.096024, 2008.11743. 
[15] A. Azatov, R. Contino, C. S. Machado and F. Riva, Helicity selection rules and noninterference for BSM amplitudes, Phys. Rev. D 95(6), 065014 (2017), doi:10.1103/PhysRevD.95.065014, 1607.05236.

[16] M. Chala, C. Krause and G. Nardini, Signals of the electroweak phase transition at colliders and gravitational wave observatories, JHEP 07, 062 (2018), doi:10.1007/JHEP07(2018)062, 1802.02168.

[17] C. W. Murphy, Dimension-8 operators in the Standard Model Eective Field Theory, JHEP 10, 174 (2020), doi:10.1007/JHEP10(2020)174, 2005.00059.

[18] T. Corbett, A. Helset, A. Martin and M. Trott, EWPD in the SMEFT to dimension eight (2021), 2102.02819.

[19] G. Panico, A. Pomarol and M. Riembau, EFT approach to the electron Electric Dipole Moment at the two-loop level, JHEP 04, 090 (2019), doi:10.1007/JHEP04(2019)090, 1810.09413 .

[20] M. Ardu and S. Davidson, What is Leading Order for LFV in SMEFT? (2021), 2103.07212 .

[21] L. Lehman, Extending the Standard Model Effective Field Theory with the Complete Set of Dimension-7 Operators, Phys. Rev. D 90(12), 125023 (2014), doi:10.1103/PhysRevD.90.125023, 1410.4193 .

[22] Y. Liao and X.-D. Ma, Renormalization Group Evolution of Dimensionseven Baryon- and Lepton-number-violating Operators, JHEP 11, 043 (2016), doi:10.1007/JHEP11(2016)043, 1607.07309.

[23] C. Hays, A. Martin, V. Sanz and J. Setford, On the impact of dimensioneight SMEFT operators on Higgs measurements, JHEP 02, 123 (2019), doi:10.1007/JHEP02(2019)123, 1808.00442.

[24] J. Ellis, S.-F. Ge, H.-J. He and R.-Q. Xiao, Probing the scale of new physics in the $Z Z \gamma$ coupling at $e^{+} e^{-}$colliders, Chin. Phys. C 44(6), 063106 (2020), doi:10.1088/1674-1137/44/6/063106, 1902.06631.

[25] S. Alioli, R. Boughezal, E. Mereghetti and F. Petriello, Novel angular dependence in Drell-Yan lepton production via dimension-8 operators, Phys. Lett. B 809, 135703 (2020), doi:10.1016/j.physletb.2020.135703, 2003.11615.

[26] J. Ellis, H.-J. He and R.-Q. Xiao, Probing new physics in dimension-8 neutral gauge couplings at $e^{+} e^{-}$colliders, Sci. China Phys. Mech. Astron. 64(2), 221062 (2021), doi:10.1007/s11433-020-1617-3, 2008.04298.

[27] C. Hays, A. Helset, A. Martin and M. Trott, Exact SMEFT formulation and expansion to $\mathcal{O}\left(v^{4} / \Lambda^{4}\right)$, JHEP 11, 087 (2020), doi:10.1007/JHEP11(2020)087, 2007.00565.

[28] J. Gu, L.-T. Wang and C. Zhang, An unambiguous test of positivity at lepton colliders (2020), 2011.03055.

[29] N. Craig, M. Jiang, Y.-Y. Li and D. Sutherland, Loops and Trees in Generic EFTs, JHEP 08, 086 (2020), doi:10.1007/JHEP08(2020)086, 2001.00017.

[30] S. Davidson, M. Gorbahn and M. Leak, Majorana neutrino masses in the renormalization group equations for lepton flavor violation, Phys. Rev. D 98(9), 095014 (2018), doi:10.1103/PhysRevD.98.095014, 1807.04283. 
[31] C. Zhang and S.-Y. Zhou, Positivity bounds on vector boson scattering at the LHC, Phys. Rev. D 100(9), 095003 (2019), doi:10.1103/PhysRevD.100.095003, 1808. 00010 .

[32] Q. Bi, C. Zhang and S.-Y. Zhou, Positivity constraints on aQGC: carving out the physical parameter space, JHEP 06, 137 (2019), doi:10.1007/JHEP06(2019)137, 1902.08977.

[33] G. N. Remmen and N. L. Rodd, Consistency of the Standard Model Effective Field Theory, JHEP 12, 032 (2019), doi:10.1007/JHEP12(2019)032, 1908.09845.

[34] G. N. Remmen and N. L. Rodd, Flavor Constraints from Unitarity and Analyticity, Phys. Rev. Lett. 125(8), 081601 (2020), doi:10.1103/PhysRevLett.125.081601, 2004. 02885.

[35] G. N. Remmen and N. L. Rodd, Signs, Spin, SMEFT: Positivity at Dimension Six (2020), 2010.04723.

[36] Q. Bonnefoy, E. Gendy and C. Grojean, Positivity bounds on Minimal Flavor Violation, JHEP 04, 115 (2021), doi:10.1007/JHEP04(2021)115, 2011.12855.

[37] B. Bellazzini, J. Elias Miró, R. Rattazzi, M. Riembau and F. Riva, Positive Moments for Scattering Amplitudes (2020), 2011.00037.

[38] B. Grzadkowski, M. Iskrzynski, M. Misiak and J. Rosiek, Dimension-Six Terms in the Standard Model Lagrangian, JHEP 10, 085 (2010), doi:10.1007/JHEP10(2010)085, 1008.4884 .

[39] H.-L. Li, Z. Ren, J. Shu, M.-L. Xiao, J.-H. Yu and Y.-H. Zheng, Complete Set of Dimension-8 Operators in the Standard Model Effective Field Theory (2020), 2005. 00008 .

[40] J. Elias-Miro, J. R. Espinosa and A. Pomarol, One-loop non-renormalization results in EFTs, Phys. Lett. B 747, 272 (2015), doi:10.1016/j.physletb.2015.05.056, 1412.7151.

[41] C. Cheung and C.-H. Shen, Nonrenormalization Theorems without Supersymmetry, Phys. Rev. Lett. 115(7), 071601 (2015), doi:10.1103/PhysRevLett.115.071601, 1505. 01844 .

[42] Z. Bern, J. Parra-Martinez and E. Sawyer, Nonrenormalization and Operator Mixing via On-Shell Methods, Phys. Rev. Lett. 124(5), 051601 (2020), doi:10.1103/PhysRevLett.124.051601, 1910.05831.

[43] F. del Aguila, M. Perez-Victoria and J. Santiago, Observable contributions of 口 new exotic quarks to quark mixing, JHEP 09, 011 (2000), doi:10.1088/11266708/2000/09/011, hep-ph/0007316.

[44] F. del Aguila, J. de Blas and M. Perez-Victoria, Effects of new leptons in Electroweak Precision Data, Phys. Rev. D 78, 013010 (2008), doi:10.1103/PhysRevD.78.013010, 0803.4008 .

[45] F. del Aguila, J. de Blas and M. Perez-Victoria, Electroweak Limits on General New Vector Bosons, JHEP 09, 033 (2010), doi:10.1007/JHEP09(2010)033, 1005.3998.

[46] J. de Blas, M. Chala, M. Perez-Victoria and J. Santiago, Observable Effects of General New Scalar Particles, JHEP 04, 078 (2015), doi:10.1007/JHEP04(2015)078, 1412. 8480. 
[47] J. de Blas, J. C. Criado, M. Perez-Victoria and J. Santiago, Effective description of general extensions of the Standard Model: the complete tree-level dictionary, JHEP 03, 109 (2018), doi:10.1007/JHEP03(2018)109, 1711.10391.

[48] A. Alloul, N. D. Christensen, C. Degrande, C. Duhr and B. Fuks, FeynRules 2.0 - A complete toolbox for tree-level phenomenology, Comput. Phys. Commun. 185, 2250 (2014), doi:10.1016/j.cpc.2014.04.012, 1310.1921.

[49] T. Hahn, Generating Feynman diagrams and amplitudes with FeynArts 3, Comput. Phys. Commun. 140, 418 (2001), doi:10.1016/S0010-4655(01)00290-9, hep-ph/ 0012260 .

[50] T. Hahn and M. Perez-Victoria, Automatized one loop calculations in four-dimensions and D-dimensions, Comput. Phys. Commun. 118, 153 (1999), doi:10.1016/S00104655(98)00173-8, hep-ph/9807565.

[51] A. Carmona, A. Lazopoulos, P. Olgoso and J. Santiago, MatchMaker: automated one-loop matching, (to appear) .

[52] V. Gherardi, D. Marzocca and E. Venturini, Matching scalar leptoquarks to the SMEFT at one loop, JHEP 07, 225 (2020), doi:10.1007/JHEP07(2020)225, [Erratum: JHEP 01, 006 (2021)], 2003.12525.

[53] J. C. Criado and M. Pérez-Victoria, Field redefinitions in effective theories at higher orders, JHEP 03, 038 (2019), doi:10.1007/JHEP03(2019)038, 1811.09413.

[54] J. Elias Miró, J. Ingoldby and M. Riembau, EFT anomalous dimensions from the S-matrix, JHEP 09, 163 (2020), doi:10.1007/JHEP09(2020)163, 2005.06983.

[55] P. Baratella, C. Fernandez and A. Pomarol, Renormalization of Higher-Dimensional Operators from On-shell Amplitudes, Nucl. Phys. B 959, 115155 (2020), doi:10.1016/j.nuclphysb.2020.115155, 2005.07129.

[56] M. E. Peskin and T. Takeuchi, A New constraint on a strongly interacting Higgs sector, Phys. Rev. Lett. 65, 964 (1990), doi:10.1103/PhysRevLett.65.964.

[57] J. de Blas, M. Ciuchini, E. Franco, S. Mishima, M. Pierini, L. Reina and L. Silvestrini, Electroweak precision constraints at present and future colliders, PoS ICHEP2016, 690 (2017), doi:10.22323/1.282.0690, 1611.05354.

[58] F. Maltoni, L. Mantani and K. Mimasu, Top-quark electroweak interactions at high energy, JHEP 10, 004 (2019), doi:10.1007/JHEP10(2019)004, 1904.05637.

[59] X.-m. Zhang, Operators analysis for Higgs potential and cosmological bound on Higgs mass, Phys. Rev. D 47, 3065 (1993), doi:10.1103/PhysRevD.47.3065, hep-ph/ 9301277.

[60] C. Grojean, G. Servant and J. D. Wells, First-order electroweak phase transition in the standard model with a low cutoff, Phys. Rev. D 71, 036001 (2005), doi:10.1103/PhysRevD.71.036001, hep-ph/0407019.

[61] D. Bodeker, L. Fromme, S. J. Huber and M. Seniuch, The Baryon asymmetry in the standard model with a low cut-off, JHEP 02, 026 (2005), doi:10.1088/11266708/2005/02/026, hep-ph/0412366. 
[62] C. Delaunay, C. Grojean and J. D. Wells, Dynamics of Non-renormalizable Electroweak Symmetry Breaking, JHEP 04, 029 (2008), doi:10.1088/11266708/2008/04/029, 0711.2511.

[63] J. de Vries, M. Postma, J. van de Vis and G. White, Electroweak Baryogenesis and the Standard Model Effective Field Theory, JHEP 01, 089 (2018), doi:10.1007/JHEP01(2018)089, 1710.04061.

[64] C. Caprini et al., Detecting gravitational waves from cosmological phase transitions with LISA: an update, JCAP 03, 024 (2020), doi:10.1088/1475-7516/2020/03/024, 1910.13125 .

[65] V. A. Kuzmin, V. A. Rubakov and M. E. Shaposhnikov, On the Anomalous Electroweak Baryon Number Nonconservation in the Early Universe, Phys. Lett. B 155, 36 (1985), doi:10.1016/0370-2693(85)91028-7.

[66] P. H. Chankowski and Z. Pluciennik, Renormalization group equations for seesaw neutrino masses, Phys. Lett. B 316, 312 (1993), doi:10.1016/0370-2693(93)90330-K, hep-ph/9306333.

[67] K. S. Babu, C. N. Leung and J. T. Pantaleone, Renormalization of the neutrino mass operator, Phys. Lett. B 319, 191 (1993), doi:10.1016/0370-2693(93)90801-N, hep-ph/9309223.

[68] S. Antusch, M. Drees, J. Kersten, M. Lindner and M. Ratz, Neutrino mass oper1 ator renormalization revisited, Phys. Lett. B 519, 238 (2001), doi:10.1016/S03702693(01)01127-3, hep-ph/0108005.

[69] R. Alonso, H.-M. Chang, E. E. Jenkins, A. V. Manohar and B. Shotwell, Renormalization group evolution of dimension-six baryon number violating operators, Phys. Lett. B 734, 302 (2014), doi:10.1016/j.physletb.2014.05.065, 1405.0486.

[70] Y. Liao and X.-D. Ma, Renormalization Group Evolution of Dimension-seven Operators in Standard Model Effective Field Theory and Relevant Phenomenology, JHEP 03, 179 (2019), doi:10.1007/JHEP03(2019)179, 1901.10302.

[71] M. Chala and A. Titov, Neutrino masses in the Standard Model effective field theory (2021), 2104.08248 .

[72] M. Jiang, T. Ma and J. Shu, Renormalization Group Evolution from On-shell SMEFT, JHEP 01, 101 (2021), doi:10.1007/JHEP01(2021)101, 2005.10261.

[73] A. Barzinji, M. Trott and A. Vasudevan, Equations of Motion for the Standard Model Effective Field Theory: Theory and Applications, Phys. Rev. D 98(11), 116005 (2018), doi:10.1103/PhysRevD.98.116005, 1806.06354.

[74] J. C. Criado, MatchingTools: a Python library for symbolic effective field theory calculations, Comput. Phys. Commun. 227, 42 (2018), doi:10.1016/j.cpc.2018.02.016, 1710.06445 . 